\title{
On the Development of Baroclinic Waves Influenced by Friction and Heating ${ }^{1}$ )
}

\author{
By A. Wiin-Nielsen, A. Vernekar and C. H. YANG ${ }^{2}$
}

\begin{abstract}
Summary - The influence of surface skin friction and a specific type of heating on the stability of baroclinic waves in a two-level, quasi-geostrophic model is investigated. It is found that the effect of friction alone changes the neutral stability curve in such a way that a broader band of wavelengths are unstable for a given value of the vertical windshear. The neutral stability curve is independent of the intensity of friction in this case. The effect of heating is to make all waves longer than a certain critical wave length unstable, but the amplification rate is very small for large values of the wavelength. The combined effect of friction and heating will in general tend to stabilize the waves. The amplification rate is investigated in all cases.

Numerical integrations of the linearized equations show that the flow in the stable case will reach a steady state characterized by vanishing dissipation and heat transport, but with a certain wave length dependent ratio between the kinetic energy of the vertical shear flow and the vertical mean flow. It is shown that this ratio can be predicted from the steady state solutions in the adiabatic, frictionless case. A comparison is made between the predictions of the energetics of the waves in the model and observational studies.
\end{abstract}

\section{Introduction}

The baroclinic stability properties of the two-level quasi-geostrophic model have been investigated in great detail as seen from studies by Eliassen [3] ${ }^{3}$ ), Phillirs [8] and many others. HoLOPAINEN [6] has studied the influence of surface friction on the growth rate of baroclinic disturbances and has shown by numerical examples that the interval of unstable wave lengths becomes somewhat broader for a given value of the vertical windshear when the effects of surface friction are included in the model. A similar conclusion was reached by HaltineR and CAVERLY [5] in their study of the same subject. The present study may be considered as an extension of the two studies mentioned above.

Although certain types of frictional processes easily can be incorporated in a stability analysis of a two-level model, it is much more difficult to include the effects of diabatic heating because several of the physical processes by which the atmosphere

1) Research supported by the Section on Atmospheric Sciences, National Science Foundation, GP-2561.

2) Department of Meteorology and Oceanography, University of Michigan. Contribution No. 99 from the Department of Meteorology and Oceanography, University of Michigan.

3) Numbers in brackets refer to References, pages 160/161. 
is heated are of a non-linear nature. As an example we may mention the role played by liberation of the heat of condensation which depends upon the vertical velocity field, the temperature field and the moisture field in a non-linear way. Studies by WIIN-NIELSEN and BRown [11] and BRown [1] have, however, indicated that the generation of eddy available potential energy on the average is negative in the troposphere. Since the generation of available potential energy depends upon the correlation of the heat sources and the temperature field we may conclude that the eddy component of the heat sources is negatively correlated with the eddy temperature field, and we may use this observational result to model the heat sources to the first degree of approximation as will be shown later in this study.

A direct application of the linear perturbation method becomes somewhat cumbersome when we are interested in the processes of friction and heating because all coefficients become complex. One can circumvent this problem by forming equations of the statistical-dynamical type which are somewhat easier to handle from a numerical point of view. Such a procedure will be used in this study. An additional advantage of using such an approach in a linear problem is that some of the energetical properties of the model easily can be understood because one of the equations expresses the rate of change of kinetic energy.

The results of our study will be compared with recent observational studies of the energetics of the atmosphere.

\section{The Equations for the Model}

The linearized equations for the quasi-geostrophic two-level model without friction and heating are derived many places in the literature (see for example THOMPSON [10]). In the present study we shall include friction and heating in the equations. Only the effect of surface friction will be considered, and we shall in this respect follow the procedure used by PHILLIPs [9]. The equations take the following form when we neglect all reference to the meridional coordinate:

$$
\begin{gathered}
\frac{\partial}{\partial t}\left(\frac{\partial^{2} \phi^{*}}{\partial x^{2}}\right)+U^{*} \frac{\partial}{\partial x}\left(\frac{\partial^{2} \phi^{*}}{\partial x^{2}}\right)+U^{\prime} \frac{\partial}{\partial x}\left(\frac{\partial^{2} \phi^{\prime}}{\partial x^{2}}\right)+\beta \frac{\partial \phi^{*}}{\partial x}=-G\left(\frac{\partial^{2} \phi^{*}}{\partial x^{2}}-2 \frac{\partial^{2} \phi^{\prime}}{\partial x^{2}}\right) \\
\frac{\partial}{\partial t}\left(\frac{\partial^{2} \phi^{\prime}}{\partial x^{2}}\right)+U^{*} \frac{\partial}{\partial x}\left(\frac{\partial^{2} \phi^{\prime}}{\partial x^{2}}\right)+U^{\prime} \frac{\partial}{\partial x}\left(\frac{\partial^{2} \phi^{*}}{\partial x^{2}}\right)+\beta \frac{\partial \phi^{\prime}}{\partial x}= \\
=\frac{f_{0}^{2}}{P} \omega+G\left(\frac{\partial^{2} \phi^{*}}{\partial x^{2}}-2 \frac{\partial^{2} \phi^{\prime}}{\partial x^{2}}\right) \\
\frac{\partial \phi^{\prime}}{\partial t}+U^{*} \frac{\partial \phi^{\prime}}{\partial x}-U^{\prime} \frac{\partial \phi^{*}}{\partial x}-\frac{\sigma P}{2} \omega=\frac{R}{2} \frac{d Q}{d t}
\end{gathered}
$$

The data levels used in the two-level model are normally the 25 and 75 cb surfaces. Any quantity marked by an asterisk is half the sum of the values at the reference levels while a quantity marked by a prime is half the difference between the corresponding 
values. $\phi=g z$ is the geopotential, $g$ gravity, $z$ height, $U$ zonal wind, $\omega$ vertical velocity (at the middle level, $50 \mathrm{cb}$ ), $f_{0}$ a standard value of the Coriolis parameter, $\beta=d f / d y$, $P=50 \mathrm{cb}$, and $\sigma=-\alpha \partial \ln \theta / \partial p$ a (constant) measure of static stability. The parameter $G$ which measures the frictional effect is defined as $G=\left(g C_{d} \varrho V_{s}\right) /(2 P)$ where $C_{d}$ is the drag coefficient, $\varrho$ density and $V_{s}$ a standard value of the surface wind. If not otherwise stated we shall use a standard value of $G=3 \times 10^{-6} \mathrm{sec}^{-1}$.

$d Q / d t$ as it appears in (2.3) is the eddy component of the diabetic heating per unit mass and unit time. It will be assumed in this study that the averaged effect of the eddy component of diabatic heating can be incorporated in the linear analysis by a relation of the type

$$
\frac{d Q}{d t}=-a_{1} \phi^{\prime}
$$

which says that the atmosphere is heated in regions where the temperature is below the zonally averaged temperature, while it is cooled where the temperature exceeds the zonal average. This heating expression is similar to those used by CHARNEY [2] and FJøRTOFT [4] in their studies of certain aspects of the general circulation. It should be stressed that an expression as (2.4) undoubtedly is an oversimplification of the heating in the atmosphere. As discussed by FJøRTOFT (loc. cit.) this is particularly so because of the role of condensation in largescale flow pattern in the atmosphere. However, (2.4) does agree with the average behavior of the atmosphere to the extent that it follows from (2.4) that the generation of eddy available potential energy $G\left(A_{E}\right)$ is negative as shown by observational studies by WIIN-NIELSEN and BROWN [11] and Brown [1]. The first of these studies can be used to obtain an estimate of the coefficient $a_{1}$ in the following way. $G\left(A_{E}\right)$ for a two-level model is of the form:

$$
G\left(A_{E}\right)=k \int_{S} \frac{d Q}{d t} \cdot \phi^{\prime} d S,
$$

where $k$ is a constant, while $d S$ is a horizontal area element. Using (2.4) we may write (2.5) in the form

$$
G\left(A_{E}\right)=-a_{1} \cdot k \int_{S} \phi^{\prime 2} d S .
$$

$G\left(A_{E}\right)$ and the integral on the right hand side of (2.6) were evaluated for the month of January 1959 by WIIN-NIELSEN and BRowN [11]. Using these numerical values and recalling that the estimates were made on the basis of 85 and $50 \mathrm{cb}$ data, while $\phi^{\prime}$ is the geopotential of a layer of $25 \mathrm{cb}$ we find that $a_{1}=2.65 \times 10^{-6} \mathrm{sec}^{-1}$. We may therefore write:

$$
\frac{R}{2 C_{p}} \frac{d Q}{d t}=-\frac{R}{2 C_{p}} a_{1} \phi^{\prime}=-a \phi^{\prime},
$$

where $a \cong 0.4 \times 10^{-6} \sec ^{-1}$. 
The perturbations which we are going to consider will satisfy the relation $\partial^{2} \phi / \partial x^{2}=-k^{2} \phi(k$ is the wave number) and similar relations for the other dependent variables. When we introduce these relations in (2.1)-(2.3) and eliminate the vertical velocity we may write the resulting equations in the form:

$$
\left.\begin{array}{c}
\frac{\partial \phi^{*}}{\partial t}+\left(U^{*}-C_{\mathrm{R}}\right) \frac{\partial \phi^{*}}{\partial x}+U^{\prime} \frac{\partial \phi^{\prime}}{\partial x}=-G\left(\phi^{*}-2 \phi^{\prime}\right) \\
\frac{\partial \phi^{\prime}}{\partial t}+\left(U^{*}-\frac{k^{2}}{k^{2}+q^{2}} C_{R}\right) \frac{\partial \phi^{\prime}}{\partial x}+ \\
\frac{k^{2}-q^{2}}{k^{2}+q^{2}} U^{\prime} \frac{\partial \phi^{*}}{\partial x}= \\
=\frac{k^{2}}{k^{2}+q^{2}} G\left(\phi^{*}-2 \phi^{\prime}\right)-\frac{q^{2}}{k^{2}+q^{2}} a \phi^{\prime}
\end{array}\right\}
$$

in which we have introduced the notations

$$
C_{R}=\frac{\beta}{k^{2}}
$$

and

$$
q^{2}=\frac{2 f_{0}^{2}}{\sigma P^{2}}
$$

It is pertinent to mention that a single equation for the vertical velocity, $\omega$, can be obtained by introducing the relation $\partial^{2} \phi^{\prime} \partial \partial x^{2}=-k^{2} \phi^{\prime}$ in (2.2) and eliminate $\partial \phi^{\prime} / \partial t$ between (2.2) and (2.3). We obtain:

$$
\omega=\frac{2}{\sigma P} \cdot \frac{k^{2}}{k^{2}+q^{2}}\left[-2 U^{\prime} \frac{\partial \phi^{*}}{\partial x}+C_{R} \frac{\partial \phi^{\prime}}{\partial x}+G\left(\phi^{*}-2 \phi^{\prime}\right)+a \phi^{\prime}\right] .
$$

\section{Statistical-dynamical Equations}

As mentioned in the introduction it is advantageous to replace the equations given in section 2 by another closed set of equations which are directly related to the changes of kinetic energy in this simple model. The kinetic energy will be subdivided into the kinetic energy of the vertical mean flow and the energy of the vertical shear flow. The kinetic energy of the vertical mean flow per unit area is:

$$
K^{*}=\frac{1}{g \lambda} \int_{0}^{p_{0}} \int_{0}^{\lambda} \frac{1}{2} v^{* 2} \quad d p \quad \frac{P}{g} \frac{k^{2}}{f_{0}^{2}} \overline{\phi^{* 2}}
$$

where we have used the geostrophic assumption and integrated by parts and where the bar denotes an average over one wavelength, i.e.

$$
\overline{()}=\frac{1}{\lambda} \int_{0}^{\lambda}() d x \text {. }
$$


We find in a similar way that the shear flow kinetic energy $K^{\prime}$ is

$$
K^{\prime}=\frac{P}{g} \frac{k^{2}}{f_{0}^{2}} \overline{\phi^{\prime 2}}
$$

while the available potential energy can be expressed in the form:

$$
A=\frac{2}{g \sigma P} \overline{\phi^{\prime 2}}
$$

which shows that the available potential energy in the linearized model is proportional to the shear flow kinetic energy for a given wave number.

The equations (3.1), (3.3) and (3.4) show clearly how we can form the energy equations for the model. To form the equation for $d K^{*} / d t$ we must multiply (2.8) by $\left(2 P k^{2}\right) /\left(g f_{0}^{2}\right) \cdot \phi^{*}$ and integrate over one wavelength. A similar procedure must be used in connection with (2.9) in order to form the equation for $d K^{\prime} / d t$. We find:

$$
\frac{d K^{*}}{d t}=2 \frac{P k^{2}}{g} \frac{U_{0}}{f_{0}} U^{\prime}-2 G\left(K^{*}-2 L\right)
$$

and

$\frac{d K^{\prime}}{d t}=-2 \frac{k^{2}-q^{2} P k^{2}}{k^{2}+q^{2}} \frac{k^{\prime}}{g} U_{0} \quad H+2 \frac{k^{2}}{k^{2}+q^{2}} G\left(L-2 K^{\prime}\right)-2 \frac{q^{2}}{k^{2}+q^{2}} a K^{\prime}$

in which we have defined the following symbols:

and

$$
L=\frac{P}{g} \frac{k^{2}}{f_{0}^{2}} \overline{\phi^{*} \phi^{\prime}}
$$

$$
H=\widehat{\phi^{\prime} v^{*}} \text {. }
$$

The function $L$ as defined has the same dimension as $K^{*}$ and $K^{\prime}$ and is measured in the unit of energy per unit area. Statistically $L$ is proportional to the covariance of $\phi^{*}$ and $\phi^{\prime}$ just as $K^{*}$ and $K^{\prime}$ are proportional to the variances of $\phi^{*}$ and $\phi^{\prime}$, respectively. Since $\phi^{\prime}$ is a measure of the mean temperature of the layer between 25 and 75 $\mathrm{cb}$ it is obvious that $H$ is a measure of the meridional transport of sensible heat in the model. The total heat transport per unit length may be written

$$
\frac{1}{g \lambda} \int_{0}^{p_{0}} \int_{0}^{\lambda} C_{p} T v d x d p=\frac{2 P}{g} C_{p} \overline{T v^{*}}=\frac{4 P}{g} \frac{C_{p}}{R} \overline{\phi^{\prime} v^{*}}
$$

In order to get the total heat transport in the unit: $\mathrm{kjm}^{-1} \mathrm{sec}^{-1}$ we must therefore multiply $H$ by $\left(4 P C_{p}\right) /(g R)=70 \mathrm{tm}^{-3}$. In the following we shall refer to $H$ as the heat transport, while $L$ which at any given time is a measure of the phase lag between $\phi^{*}$ and $\phi^{\prime}$ will be called the lag function. 
Considering the results obtained in (3.5) and (3.6) it is now obvious that if we want to form a closed system of equations we must derive equations for $d L / d t$ and $d H / d t$. These equations are formed from (2.8) and (2.9) guided by the definitions (3.7) and (3.8). After some rearrangement we can write the equations in the form

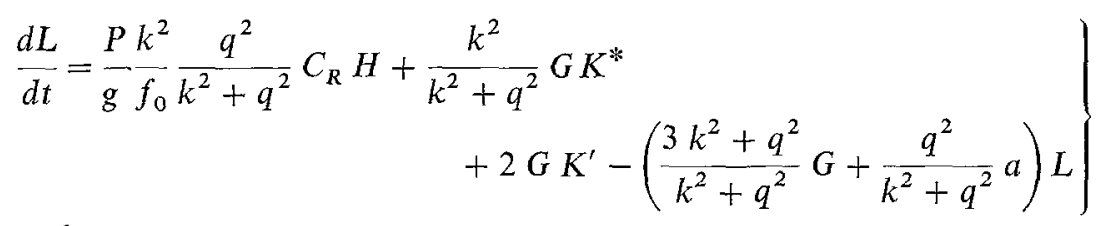

and

$$
\left.\begin{array}{rl}
\frac{d H}{d t}=-\frac{g}{P} f_{0} \frac{q^{2}}{k^{2}+q^{2}} C_{R} L+\frac{g}{P} f_{0} U^{\prime} & K^{\prime}-\frac{g}{P} f_{0} \frac{k^{2}-q^{2}}{k^{2}+q^{2}} U^{\prime} K^{*} \\
& -\left(\frac{3 k^{2}+q^{2}}{k^{2}+q^{2}}-G+\frac{q^{2}}{k^{2}+q^{2}} a\right) H
\end{array}\right\}
$$

The equations (3.5), (3.6), (3.10) and (3.11) form a closed system in the variables $K^{*}, K^{\prime}, L$ and $H$. For given values of $U^{\prime}, G$ and $a$ and a given wave number $k$ we can in principle solve the equations. If it turns out to be impossible to solve the equations by standard methods one can always solve them numerically as an initial value problem.

\section{Energy Conversion Relations}

The general energy relations for the two level model have been treated by many authors, see for example PHILliPs [9]. In this study we shall consider the kinetic energy of the vertical shear flow separately (WIIN-NIELSEN [12] and WIIN-NIELSEN and DRAKE [15]). With reference to these papers we find that the symbolic energy equations for the eddy components of the present model are:

$$
\begin{aligned}
\frac{d K^{*}}{d t} & =C\left(K^{\prime}, K^{*}\right)-D\left(K^{*}\right) \\
\frac{d K^{\prime}}{d t} & =C\left(A, K^{\prime}\right)-C\left(K^{\prime}, K^{*}\right)+D\left(K^{\prime}\right) \\
\frac{d A}{d t} & =C\left(A_{Z}, A\right)-C\left(A, K^{\prime}\right)+G_{e}(A)
\end{aligned}
$$

$A_{\mathrm{Z}}$ denotes the available potential energy in the zonal flow, while $K^{*}, K^{\prime}$ and $A$ are the energies in the eddy. Using the basic equations (2.3), (2.8) and (2.9) we can derive the expressions for all energy processes in the model. They are:

$$
C\left(A_{Z}, A\right)=\frac{2 P}{g} \frac{q^{2}}{f_{0}} U^{\prime} H
$$




$$
\begin{gathered}
C\left(A, K^{\prime}\right)=\frac{4 P k^{2}}{g} \frac{q^{2}}{f_{0} k^{2}+q^{2}} U^{\prime} H+2 \frac{q^{2}}{k^{2}+q^{2}} G\left(2 K^{\prime}-L\right)-2 \frac{q^{2}}{k^{2}+q^{2}} a K^{\prime} \\
C\left(K^{\prime}, K^{*}\right)=\frac{2 P \frac{k^{2}}{g} \frac{f_{0}}{f_{0}} U^{\prime} H}{G_{e}(A)=-2 a A=-2 \frac{q^{2}}{k^{2}} a K^{\prime}} \\
D\left(K^{\prime}\right)=2 G\left(2 K^{\prime}-L\right) \\
D\left(K^{*}\right)=2 G\left(K^{*}-2 L\right)
\end{gathered}
$$

The general energy diagram corresponding to the model has been discussed by WIIN-NIELSEN [14]. Fig. 1 shows the diagram with solid lines indicating the energy conversions which are present in the linearized model while dashed lines show the conversions which would be present in a more general formulation.

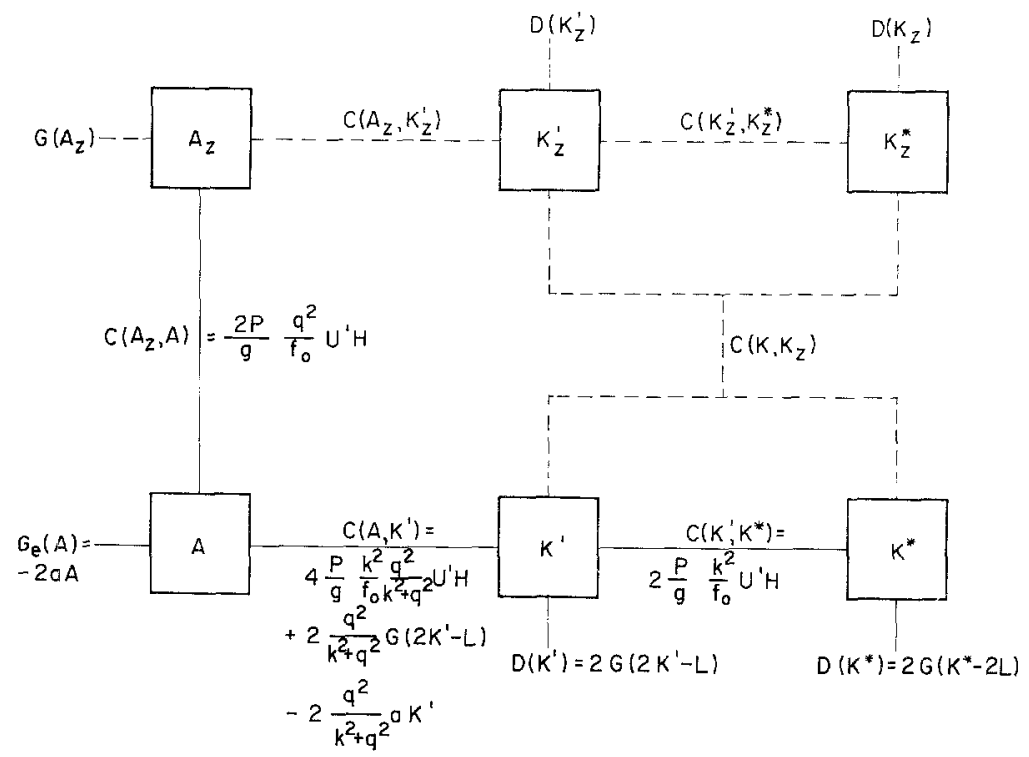

Figure 1

Schematic diagram showing the energetics of atmospheric flow. Solid lines show energy conversions present in a linear model together with the formulas for the conversions, while dashed lines indicate conversions which would be present in a general model

It is instructive to consider the energy conversion in the absence of friction $(G=0)$ and heating $(a=0)$. In this case we notice that the rate of change of $K^{*}, K^{\prime}$ and $L$ are proportional to the heat transport $H$ as can be seen from equations (3.5), (3.6) and (3.10). All energy conversions will also be proportional to $H$ as seen from (4.4)-(4.6) while $D\left(K^{*}\right)=D\left(K^{\prime}\right)=G_{e}(A)=0$. If $H>0$ we will simultaneously have energy conversions from $A_{\mathrm{Z}}$ to $A$, from $A$ to $K^{\prime}$ and from $K^{\prime}$ to $K^{*}$ while all conversions will go 
in the opposite direction if $H<0$. The conversions will however not be equal to each other. We find the following ratios between them

$$
\begin{aligned}
\frac{C\left(A, K^{\prime}\right)}{C\left(A_{Z}, A\right)} & =\frac{2 k^{2}}{k^{2}+q^{2}} \gtreqless 1, \text { if } k^{2} \gtreqless q^{2} \\
\frac{C\left(K^{\prime}, K^{*}\right)}{C\left(A, K^{\prime}\right)} & =\frac{k^{2}+q^{2}}{2 q^{2}} \gtreqless 1, \quad \text { if } k^{2} \gtreqless q^{2} .
\end{aligned}
$$

These relations show for example that if the wavelength is sufficiently small $(k>q)$ there will be an accumulation of energy in $K^{*}$ if the heat transport is positive and stays positive. The key quantity is therefore the magnitude and sign of $H$. In the more general case when $G \neq 0$ and $a \neq 0$ we will obtain more complicated expressions corresponding to (4.10) and (4.11) because the vertical velocity is influenced by heating and friction. However, we obtain in all cases the simple relation

$$
\frac{C\left(K^{\prime}, K^{*}\right)}{C\left(A_{Z}, A\right)}=\frac{k^{2}}{q^{2}}
$$

\section{Stability Analysis}

In this section we shall describe the results of a stability analysis of the model. The main purpose of the analysis is to discuss the modifications of stability caused by the influence of friction and heating. The results in the adiabatic and frictionless case are naturally well known from earlier studies, but it is advantageous to use this case as a case of comparison. The equations which are to be solved are (3.5), (3.6), (3.10) and (3.11). We shall consider solution of the form

$$
K^{*}=e^{v t} K_{0}^{*} \text {, }
$$

where $K_{0}^{*}$ is the inital value of $K^{*}$ and similar expressions for the variables $K^{\prime}, L$ and $H$. If $v$ is real and positive or complex with a positive real part we have instability.

The different cases will be treated separately below.

5A. $G=0, a=0$

In this case we can derive a single equation for $H$ by differentiating (3.11) with respect to time and substitute from (3.5), (3.6) and (3.10). The equation takes the form

$$
\frac{\mathrm{d}^{2} H}{\mathrm{~d} t^{2}}+m H=0
$$

where

$$
m=\frac{4 k^{2}\left(k^{2}-q^{2}\right)}{k^{2}+q^{2}} U^{\prime 2}+\frac{k^{2} q^{4}}{\left(k^{2}+q^{2}\right)^{2}} C_{R}^{2}
$$

Substituting $H=e^{v t} H_{0}$ we find

$$
= \pm \sqrt{-m}
$$


The neutral curve is therefore $m=0$, while $m>0$ represents $v= \pm i \sqrt{m}$ giving stable solutions of the form $\cos (s t)$ and $\sin (s t), s^{2}=m$. On the other hand, if $m<0, v$ is real and one value is positive giving instability, and the solutions are of the exponential type.

Let us briefly consider the complete solution in the stable case $m>0$. The general solution to $(5.2)$ is

$$
H=C_{1} \cos (s t)+C_{2} \sin (s t)
$$

where $C_{1}$ and $C_{2}$ are determined by the initial conditions. We find immediately $C_{1}=H_{0}$. From the initial values of $K_{0^{\prime}}^{*}, K_{0^{\prime}}^{\prime}, L_{0}$ and $H_{0}$ we can compute $H_{1}=(d H / d t)_{0}$ from (3.11). $H_{1}$ may therefore be considered as known. $C_{2}$ is now determined by differentiating (5.5) with respect to time and setting $t=0$. We find $C_{2}=1 / s H_{1}$ and consequently

$$
H=H_{0} \cos s t+\frac{1}{s} H_{1} \sin s t .
$$

The functions $K^{*}(t), K^{\prime}(t)$ and $L(t)$ are obtained from an integration of (3.5), (3.6) and (3.10) with respect to time.

The unstable case $m=-s_{*}^{2}<0$ can be treated by replacing $s$ by $s_{*}$ and sin and cos by $\sinh$ and $(-\cosh )$, respectively.

\section{B. $G \neq 0, a=0$}

Before discussing certain aspects of the solution in this case it is worthwhile to discuss the justification of introducing friction in the model but neglecting the heat sources. Friction will in general work as a dissipative force. There must therefore in general be an energy source which in the present case is the basic zonal flow which is characterized by the two constant zonal winds $U^{*}$ and $U^{\prime}$. The vertical wind shear, $U^{\prime}$, corresponds to a certain meridional temperature gradient which is equivalent to a certain amount of zonal available potential energy which constitutes the energy source for the model.

With $G \neq 0$ we can not in an easy way reduce the four basic equations to a single equation in one dependent variable. However, when the expressions (5.1) are introduced in the equations we find four homogeneous linear equations in the variables $K_{0^{\prime}}^{*}, K_{0^{\prime}}^{\prime}, L_{0}$ and $H_{0}$. The condition for non-trivial solutions to these equations is that the determinant is zero. The determinant takes the following form:

$$
\left|\begin{array}{llll}
v+2 G & 0 & -4 G & -2 U^{\prime} \frac{k^{2} P}{f_{0}} \frac{P}{g} \\
0 & v+4 \frac{k^{2}}{k^{2}+q^{2}} G & -2 \frac{k^{2}}{k^{2}+q^{2}} G & 2 U^{\prime} \frac{k^{2}}{f_{0}} \frac{P k^{2}-q^{2}}{k^{2}+q^{2}} \\
-\frac{k^{2}}{k^{2}+q^{2}} G & -2 G & v+\frac{3 k^{2}+q^{2}}{k^{2}+q^{2}} G & -C_{R} \frac{k^{2}}{f_{0}} \frac{q^{2}}{k^{2}+q^{2}} \\
\frac{g}{P} f_{0} \frac{k^{2}-q^{2}}{k^{2}+q^{2}} U^{\prime} & -\frac{g}{P} f_{0} U^{\prime} & \frac{g}{P} f_{0} \frac{q^{2}}{k^{2}+q^{2}} C_{R} & v+\frac{3 k^{2}+q^{2}}{k^{2}+q^{2}} G
\end{array}\right|=0
$$


It is seen that (5.7) leads to a fourth degree equation in $v$ with real coefficients. This equation can easily be solved by standard numerical techniques, and such solutions will be described later in the paper. We shall however first consider the neutral curve $v=0$. On this curve the kinetic energy will remain constant. It is naturally possible that the condition $v=0$ might separate two regions of stability, and that the stability boundary may occur for some finite imaginary part of $v$. In order to exclude this possibility we have derived the frequency equation directly from (2.8) and (2.9). We obtain in this case a second degree equation with complex coefficients for the phase speed. The solution of this equation leads to results which agree exactly with those given below.

The determinant (5.7) can be evaluated directly although the calculation is laborious. After suitable reduction we can write (5.7) with $v=0$ in the form

$$
q^{2}\left(q^{2} U_{n}^{\prime}+k^{2} C_{R}\right)\left(\left(3 k^{2}-5 q^{2}\right) U_{n}^{\prime}+2 q^{2} C_{R}\right)=0
$$

where $U_{n}^{\prime}$ denotes the vertical windshear corresponding to neutral conditions. We note that (5.8) does not contain the parameter $G$ which measures the intensity of friction in the model. This fact may at first seem surprising, but a substitution of (5.8) into the equations (3.5), (3.6), (3.10) and (3.11) with the time derivatives equal to zero leads to four homogeneous linear equations which we can solve apart from an arbitrary constant. It turns out that the solution may be written in the following form

$$
H=0, \frac{L}{K^{*}}=\frac{1}{2}, \frac{K^{\prime}}{K^{*}}=\frac{1}{4} .
$$

The neutral disturbances are therefore characterized by a vanishing heat transport and an amplitude ratio $a^{\prime} \mid a^{*}=\frac{1}{2}$, where $a^{\prime}$ is the amplitude of the wave in $\phi^{\prime}$, while $a^{*}$ is the amplitude of the wave in $\phi^{*}$. It follows from the ratio $a^{\prime} / a^{*}=\frac{1}{2}$ that the wave amplitude vanishes at the ground, and that $D\left(K^{*}\right)$ and $D\left(K^{\prime}\right)$ therefore are zero in the neutral case. This fact is consistent with the remark made above that $G$ does not appear in (5.8).

The solutions to (5.8) may be written in the following form:

$$
U_{n_{1}}^{\prime}=\frac{2 q^{2}}{5 q^{2}-3 k^{2}} C_{R}
$$

and

$$
U_{n_{2}}^{\prime}=-\frac{k^{2}}{q^{2}} C_{R}=-\frac{\beta}{q^{2}}
$$

The solution (5.11) corresponds to a constant negative value of the vertical windshear or in other words a decrease of wind with height. The other neutral curve given in (5.10) should be compared with the neutral curve for the adiabatic and frictionless case derived from (5.3) by setting $m=0$. We find

$$
U_{n}^{\prime}= \pm \frac{1}{2} \frac{q^{2}}{\sqrt{q^{4}-k^{4}}} C_{R}
$$


A comparison of (5.10) and (5.12), using the positive value, shows that

$$
U_{n}^{\prime}>U_{n_{1}}^{\prime} \text { for all values of } k \text {, }
$$

which indicates that the effect of friction expands the region of instability in the plane with the wavelength as abscissa and the vertical windshear as ordinate. This is furthermore indicated by the fact that (5.12) has an asymptote at $k_{a}=q$ or $\lambda_{a}=2 \pi / q \approx 3142$ $\mathrm{km}$ when $q=2 \times 10^{-6} \mathrm{~m}^{-1}$, while (5.10) has its asymptote at $k_{a}=q \sqrt{\frac{5}{3}}$ or $\lambda_{a} \approx 2430 \mathrm{~km}$.

It should be pointed out that although the numerical value of $G$ has no influence on the position of the neutral curves (5.10) and (5.11), friction will naturally influence the growth rate of the waves in the unstable region and the development of the waves in the stable region. We shall later investigate a number of these cases and, in particular, look at the asymptotic states.

5C. $G=0, a \neq 0$.

In this case we are including heating in the model, but are neglecting friction. We shall again look for solutions of the type (5.1). When these solutions are substituted in (3.5), (3.6), (3.10) and (3.11) we arrive at the following determinant equivalent to (5.7):

$$
\begin{aligned}
& v \quad \begin{array}{lll}
0 & 0 & -2 \frac{P}{g} \cdot \frac{k^{2}}{f_{0}} U^{\prime}
\end{array}
\end{aligned}
$$

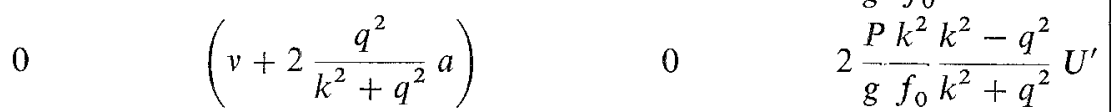

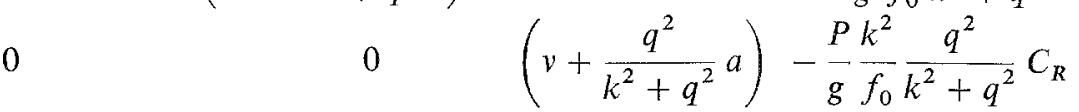

$$
\begin{aligned}
& { }_{P}^{g} f_{0} \frac{k^{2}-q^{2}}{k^{2}+q^{2}} U^{\prime} \quad-\frac{g}{P} f_{0} U^{\prime} \quad \quad g f_{0} \frac{q^{2}}{k^{2}+q^{2}} C_{R} \quad\left(v+\frac{q^{2}}{k^{2}+q^{2}} a\right)
\end{aligned}
$$

We shall again first investigate the case $v=0$ corresponding to neutral waves. An evaluation of (5.13) in this case leads to the expression

$$
\frac{k^{2}-q^{2}}{k^{2}+q^{2}} \cdot U^{\prime 2}=0,
$$

which is satisfied when $k_{a}=q$, i.e. $\lambda_{a}=3142 \mathrm{~km}$ and for $U^{\prime}=0$. A closer inspection leads to the conclusion that instability occurs whenever $\lambda>\lambda_{a}$. We find again that the neutral curve is independent of the intensity of the heating, but that the parameter $a$ will influence the growth rate in the unstable region.

5D. $G \neq 0, a \neq 0$.

The case in which both heating and friction are included is more complicated than any of the preceding cases. The determinant which determines the eigenvalues is 
simply the sum of (5.7) and (5.13). When we look for the neutral curve, i.e. $v=0$, we get a rather complicated expression $U_{n}^{\prime}=U_{n}^{\prime}(k)$ which however can be solved numerically. The position of the neutral curve will in this case depend on both $G$ and $a$. If $a$ is very small we obtain a curve which is close to the neutral curve treated in subsection 5B, while very small values of $G$ result in a neutral curve closer to the one found in subsection $5 \mathrm{C}$.

$5 E$. In this subsection we shall first consider fig. 2 which summarizes some of the results obtained in the preceding subsections. The solid curve in fig. 2 is the curve of neutral stability in the case of no heating and friction determined by $m=0$ in eq. (5.3).

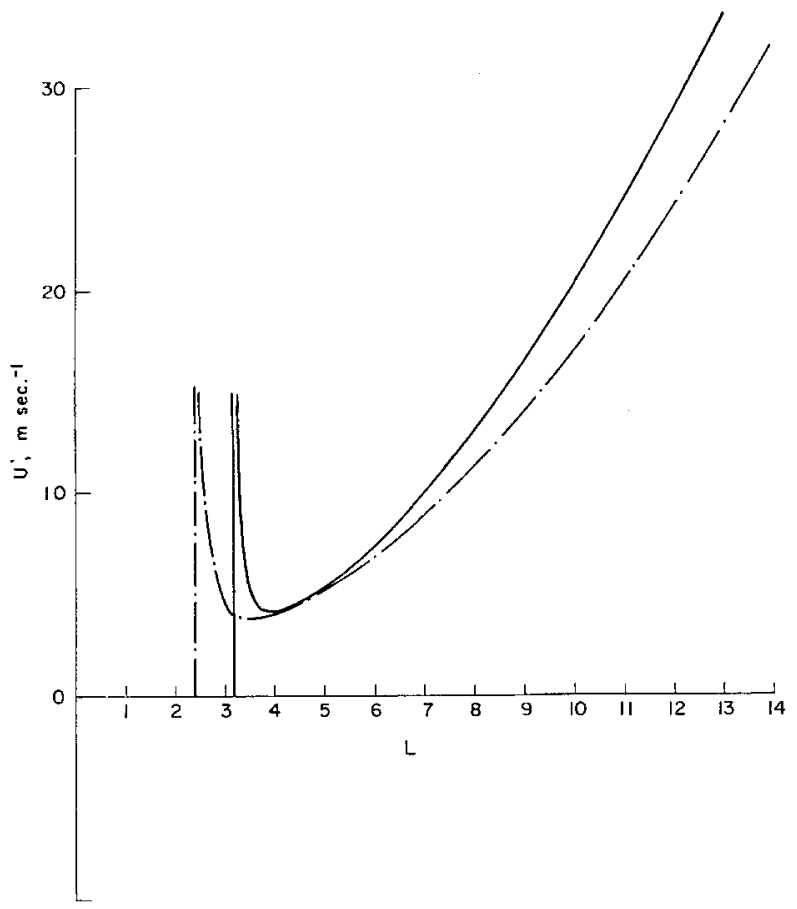

Figure 2

Neutral stability curves for frictionless model (solid curve) and model with friction (dash-dotted). The abscissa is the wavelength measured in thousands of kilometers and the ordinate is $U^{\prime}$ in $m \mathrm{sec}^{-1}$

The dash-dotted curve is the curve of neutral stability including friction, but not heating as discussed in subsection 5B and determined by eq. (5.10). The vertical asymptote in solid line is determined by $\lambda_{a}=3142 \mathrm{~km}$ while the dash-dotted asymptote corresponds to $\lambda_{a}=2430 \mathrm{~km}$. The complete region to the right of the solid asymptote represents the unstable region when we include heating, but not friction $(5 \mathrm{C})$. The somewhat larger region of instability when friction is included is in agreement with the partial results obtained by HoLOPAINEN [6]. 
When both heating and friction are included (5D) we find that the position of the neutral curve depends on the numerical values of $G$ and $a$. Some examples are shown in fig. 3. The dash-dotted curve is the curve of neutral stability in the case of friction, but no heating reproduced from fig. 2 . The thin solid curve is obtained by using the

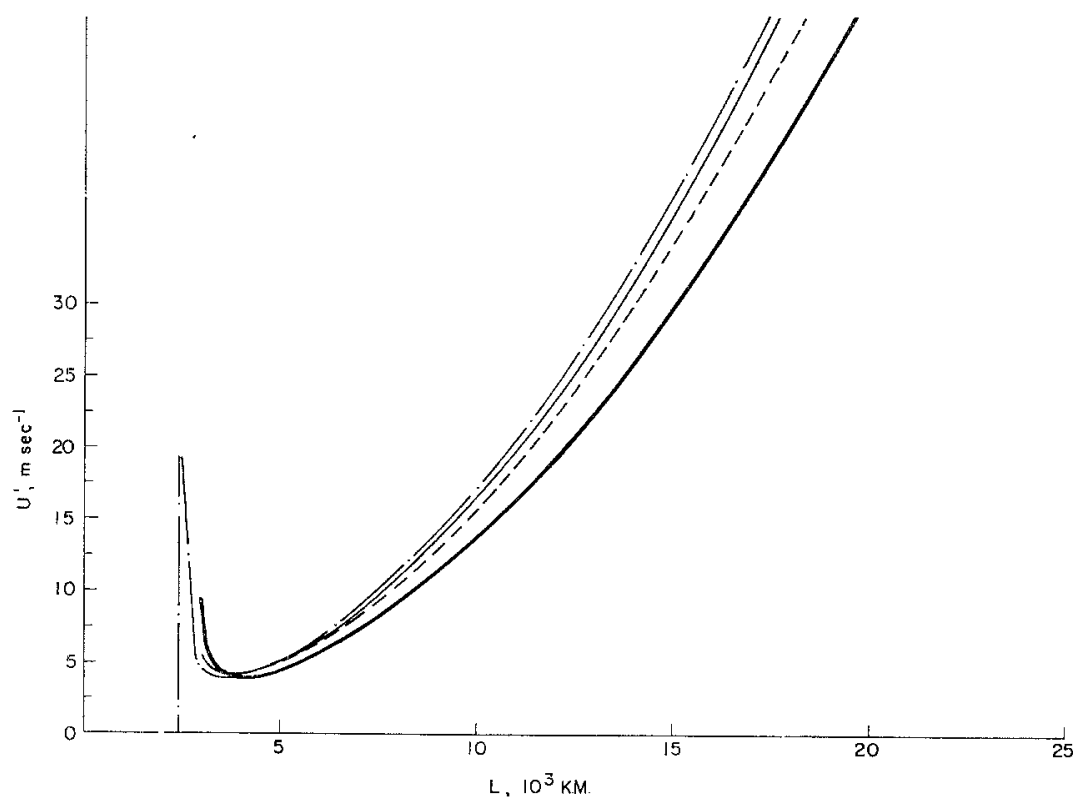

Figure 3

Neutral stability curves for various values of friction and heating. Dash-dotted line: $G \neq 0, a=0$. Thin solid line: $G=3 \times 10^{-6} \mathrm{sec}^{-1}, a=0.4 \times 10^{-6} \mathrm{sec}^{-1}$. Dashed line: $G=1 \times 10^{-6} \mathrm{sec}^{-1}, a=0.4 \times 10^{-6}$ $\sec ^{-1}$. Heavy solid line: $G=1 \times 10^{-6} \mathrm{sec}^{-1}, a=1.2 \times 10^{-6} \mathrm{sec}^{-1}$

numerical values $G=3 \times 10^{-6} \mathrm{sec}^{-1}$ and $a=0.4 \times 10^{-6} \mathrm{sec}^{-1}$ as estimated in section 2 of this paper. In view of the fact that these numerical values may be rather inaccurate it becomes of interest to investigate the changes in the neutral curve when we vary the values of $G$ and $a$. The dashed curve in fig. 3 was obtained by decreasing the value of $G$ to $1 \times 10^{-6} \mathrm{sec}^{-1}$, while $a=0.4 \times 10^{-6} \mathrm{sec}^{-1}$. Finally the thick solid line in fig. 3 corresponds to $G=1 \times 10^{-6}$ and $a=1.2 \times 10^{-6} \mathrm{sec}^{-1}$. It is seen that increasing values of $a$ and decreasing values of $G$ shift the neutral curve toward longer wavelengths.

We shall conclude this section by considering the amplification rate for the different cases. Fig. 4 shows $v^{-1}$ in the unit of days for the case $G=3 \times 10^{-6} \mathrm{sec}^{-1}$, $a=0$. In the case $G=0, a=0.4 \times 10^{-6} \mathrm{sec}^{-1}$ we found that all waves with a wavelength $\lambda>3142 \mathrm{~km}$ are unstable. Fig. 5 shows the amplification rates in this case. It is seen that only minor modifications take place in the region of maximum instability as illustrated by the curve $v^{-1}=0.2$ days. The region corresponding to rather long waves and moderate vertical windshears represents only weak instabilities having 


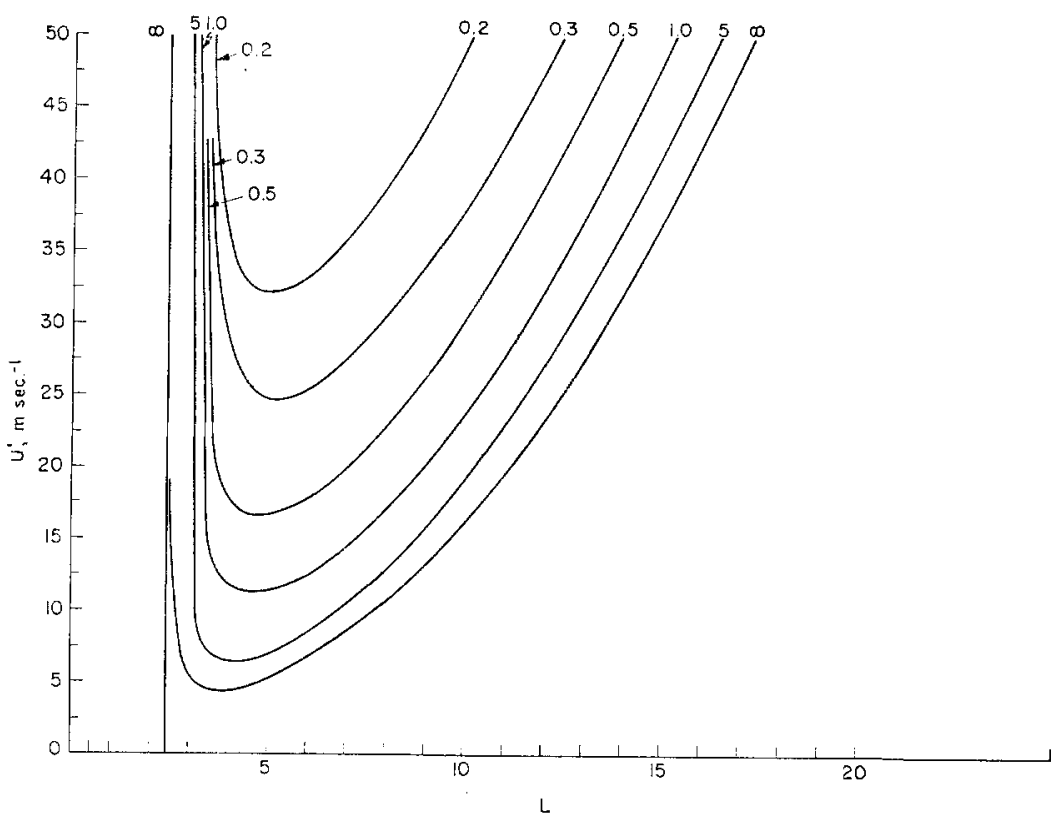

Figure 4

Stability diagram where friction is included but heating excluded in the model, $G=3 \times 10^{-6} \mathrm{sec}^{-1}$. The curves are the isolines of $v^{-1}$ in the unit: days

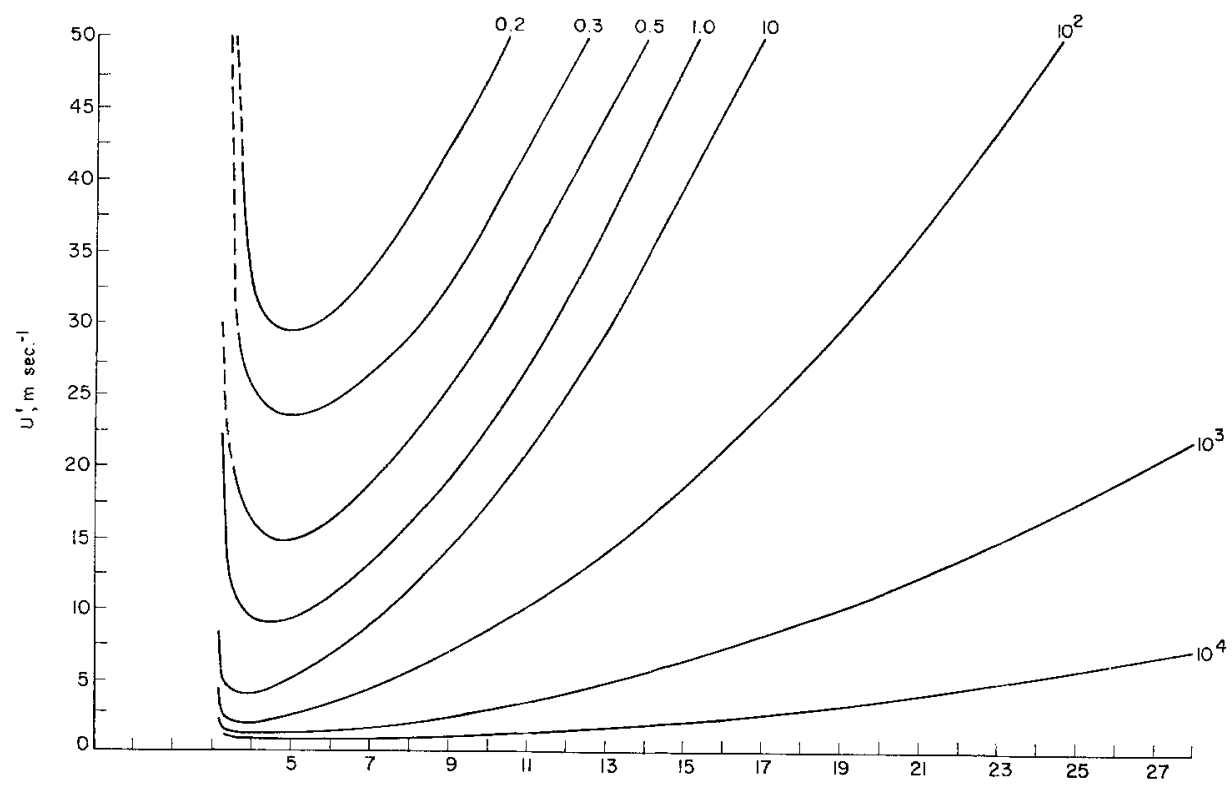

Figure 5

Stability diagram where heating is included, but friction excluded in the model, $a=0.4 \times 10^{-6} \mathrm{sec}^{-1}$. Arrangement as in fig. 4 
$e$-folding times larger than 10 days. When heating and friction both are included we find amplification rates as shown in fig. 6 . The great similarity between figs. 4 and 6 shows that the destabilizing effect of the type of heating included in this model is a minor one.

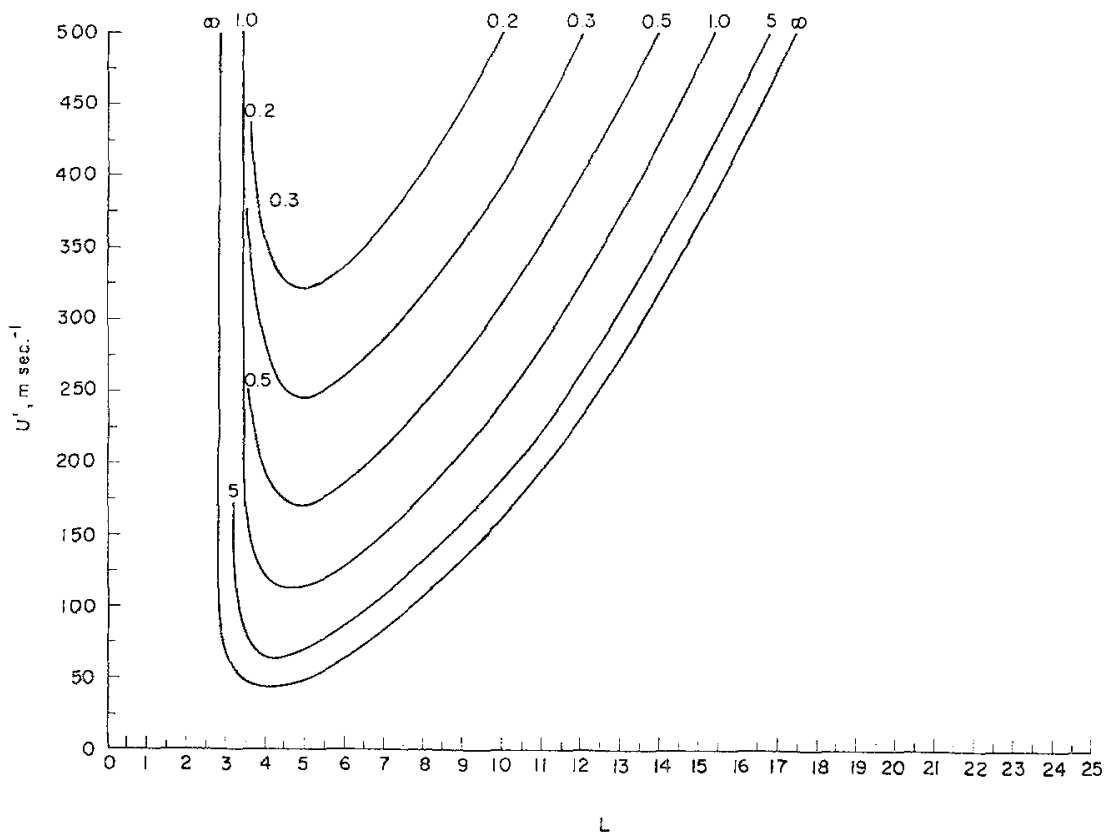

Figure 6

Stability diagram with heating and friction included. $G=3 \times 10^{-6} \mathrm{sec}^{-1}, a=0.4 \times 10^{-6} \mathrm{sec}^{-1}$. Arrangement as in fig. 4

Due to the great similarity in the shape of the curves in fig. 4 and fig. 6 it becomes difficult to make comparisons between the amplification rates. In order to illustrate the differences we have given the values of $v^{-1}$ for various values of $U^{\prime}$ for $\lambda=5000$ $\mathrm{km}$ in Table 1. A comparison of the second and first row in Table 1 shows the damping influence of friction arnounting to $25-30 \%$ for small values of $U^{\prime}$, but negligible amounts for large values of the windshear. The third row $(G=0, a \neq 0)$ as compared

Table 1

\begin{tabular}{lrlllllllll}
\hline$U^{\prime}$ & 5 & 10 & 15 & 20 & 25 & 30 & 35 & 40 & 45 & 50 \\
\hline$v^{-1}(G=0, a=0)$ & - & 0.83 & 0.50 & 0.37 & 0.29 & 0.24 & 0.20 & 0.18 & 0.16 & 0.14 \\
$v^{-1}(G \neq 0, a=0)$ & - & 1.11 & 0.63 & 0.42 & 0.32 & 0.26 & 0.22 & 0.19 & 0.17 & 0.15 \\
$v^{-1}(G=0, a \neq 0)$ & 11.4 & 0.89 & 0.52 & 0.37 & 0.29 & 0.24 & 0.21 & 0.18 & 0.16 & 0.14 \\
$v^{-1}(G \neq 0, a \neq 0)$ & - & 1.25 & 0.64 & 0.43 & 0.33 & 0.27 & 0.22 & 0.19 & 0.17 & 0.15 \\
\hline
\end{tabular}

Values of $v^{-1}$ in days in the cases $G=0, a=0, G \neq 0, a=0$, and $G=0, a \neq 0$ for various wind shears with $L=5000 \mathrm{~km}\left(G=3 \times 10^{-6} \mathrm{sec}^{-1}\right.$ and $\left.a=1.2 \times 10^{-6} \mathrm{sec}^{-1}\right)$ 
to the first row illustrates that although heating results in a larger region of instability, the amplification rates are smaller than those in the case $G=0, a=0$.

A similar comparison of amplification rates can be made for a constant value of $U^{\prime}$, but for various values of the wavelength. Table 2 shows $v^{-1}$ in days corresponding to the value $U^{\prime}=20 \mathrm{~m} \mathrm{sec}^{-1}$. We notice that the broadening of the band of unstable wavelengths is indicated in the table together with the decrease in the instability caused by friction and heating.

Table 2

\begin{tabular}{lrrrrrrrrrrrrr}
\hline$l$ & 3 & 4 & 5 & 6 & 7 & 8 & 9 & 10 & 11 & 12 & 13 & 14 \\
\hline$v^{-1}(G=0, a=0)$ & & & & 0.38 & 0.37 & 0.40 & 0.45 & 0.56 & 0.83 & - & - & - & - \\
$\nu^{-1}(G \neq 0, a=0)$ & 5.96 & 0.45 & 0.42 & 0.45 & 0.53 & 0.67 & 1.00 & 2.90 & - & - & - & - \\
$v^{-1}(G=0, a \neq 0)$ & & 0.40 & 0.37 & 0.41 & 0.48 & 0.60 & 0.89 & 3.68 & 14.72 & 26.95 & 44.35 & 64.65 \\
$v^{-1}(G \neq 0, a \neq 0)$ & 9.07 & 0.48 & 0.43 & 0.47 & 0.56 & 0.71 & 1.07 & 2.68 & 61.05 & - & - & - \\
\hline
\end{tabular}

Values of $v^{-1}$ in days as a function of wavelength for $U^{\prime}=20 \mathrm{~m} \mathrm{sec}^{-1} .\left(G=3 \times 10^{-6} \mathrm{sec}^{-1}, a=1.2 \times 10^{-6}\right.$ $\left.\sec ^{-1}\right)$

\section{Variations in Time of the Waves}

The solutions obtained in the preceding sections are particular solutions of a specified form. A more direct approach is a numerical integration with respect to time treating the problem as an initial value problem. Such integrations have been carried out in some cases including friction, but not heating. As seen from the stability analysis in section 5 , in particular a comparison between figs. 4 and 6 , heating will only give minor changes in the stability diagram. We have therefore decided to exclude heating from the numerical integrations.

We shall first consider the variations in time of the waves in the case of no friction. Since we know the exact solution to the linearized equations in this case it is a simple matter to tabulate the solutions. In each case it is necessary to define the initial state of the system. All calculations have been carried out using the following values of the parameters: $f_{0}=10^{-4} \mathrm{sec}^{-1}, g=9.807 \mathrm{~m} \mathrm{sec}^{-2}, P=50 \mathrm{cb}, \beta=16 \times 10^{-12} \mathrm{~m}^{-1} \mathrm{sec}^{-1}$ and $q=2 \times 10^{-6} \mathrm{~m}^{-1}$. In addition to the constants it is necessary to specify a value of the vertical windshear $U^{\prime}$. We have used $U^{\prime}=20 \mathrm{~m} \mathrm{sec}^{-1}$ which corresponds to a value of $d U / d z=4 \mathrm{~m} \mathrm{sec}^{-1} \mathrm{~km}^{-1}$, a value characteristic of the troposphere in winter.

It is seen from (3.5), (3.6), (3.10) and (3.11) that initial values must be given for $K^{*}, K^{\prime}, L$ and $H$. The calculations were carried out with $K^{*}=100 \mathrm{kjm}^{-2}$ and $K^{\prime}=50 \mathrm{kjm}^{-2}$. These values were selected on the basis of the spectra presented by WIIN-NIELSEN and DRAKe [15] for the kinetic energy of the vertical mean flow and the vertical shear flow as characteristic of the energy level per wave number. The definitions of $L$ and $H$ show that $L_{0}$ and $H_{0}$ can not be selected independent of each other. The reason is that $\overline{\phi^{\prime} v^{*}}$ and $\overline{\phi^{\prime} \phi^{*}}$ are intimately connected. The initial values of $L$ and $H$ were therefore selected in the following way. Knowing $K_{0}^{*}$ and $K_{0}^{\prime}$ we can 
determine the amplitude of the geopotentials $\phi^{*}$ and $\phi^{\prime}$ by the formulas

and

$$
a^{*}=\left[\frac{2 g f_{0}^{2}}{P k^{2}} K_{0}^{*}\right]^{1 / 2}
$$

$$
a^{\prime}=\left[\frac{2 g f_{0}^{2}}{P k^{2}} K_{0}^{\prime}\right]^{1 / 2}
$$

assuming that $\phi^{*}$ and $\phi^{\prime}$ are defined by

and

$$
\phi^{*}=a^{*} \cos k x
$$

$$
\phi^{\prime}=a^{\prime} \cos (k x+\gamma)
$$

where $\gamma$ is the phase difference which determines how much the thermal field $\phi^{\prime}$ is lagging behind the field $\phi^{*}$. It is furthermore seen from (6.3) and (6.4) that

$$
\overline{\phi^{*} \phi^{\prime}}=\frac{1}{2} a^{*} a^{\prime} \cos \gamma
$$

and

$$
\overline{\phi^{\prime} v^{*}}=\frac{1}{2} \frac{k}{f_{0}} a^{*} a^{\prime} \sin \gamma .
$$

If we therefore make a choice of $\gamma$ initially we can evaluate $L_{0}$ and $H_{0}$ from the formulas

and

$$
L_{0}=\frac{P k^{2}}{g} \frac{1}{f_{0}} \frac{1}{2} a^{*} a^{\prime} \cos \gamma
$$

$$
H_{0}=\frac{1}{2} \frac{k}{f_{0}} a^{*} a^{\prime} \sin \gamma
$$

We shall first describe a particularly simple case in which $G=0$ and $\gamma=0$. We have thus $H_{0}=0$. In describing the calculations we must distinguish between the stable and unstable solutions. The calculations in section 5 show that the waves will be unstable for wavelength between $3150 \mathrm{~km}$ and $9810 \mathrm{~km}$, or if we measure the wavelength in $10^{6} \mathrm{~m}$ for

$$
3.15 \leqq l \leqq 9.81
$$

Fig. 7a shows the variation of the heat transport for the stable waves, i.e. $l=1,2$ and 3 and $l=10,11,12,13$, and 14. The wavelength $l$ is given on the left side of the figure while the corresponding period $T$ measured in days is given on the right side. We notice immediately a difference in the behavior of the heat transport. For $l=2,3$ and $10, H$ is increasing in the beginning while an initial decrease is found in the other cases. This initial tendency can easily be verified from (3.10) by asking when 
$(d H / d t)_{0}>0$. It turns out that the inequality is satisfied if

in agreement with fig. 7a.

$$
1.85 \leqq l \leqq 10.95
$$

Figs. $7 \mathrm{~b}$ and $7 \mathrm{c}$ show the variation over one period of $K^{*}$ and $K^{\prime}$, respectively. A comparison of fig. $7 \mathrm{a}$ and $7 \mathrm{c}$ shows that the mean flow kinetic energy increases initially if the heat transport increases at $t=0$. This means that the kinetic energy will accumulate initially in the reservoir $K^{*}$. On the other hand, a comparison of fig. 7b and fig. 7c shows that the variation of $K^{\prime}$ and $K^{*}$ through one period is such that $K^{\prime}$ and $K^{*}$ are in phase for the long stable waves $(l \geqq 10)$ while they are exactly out of

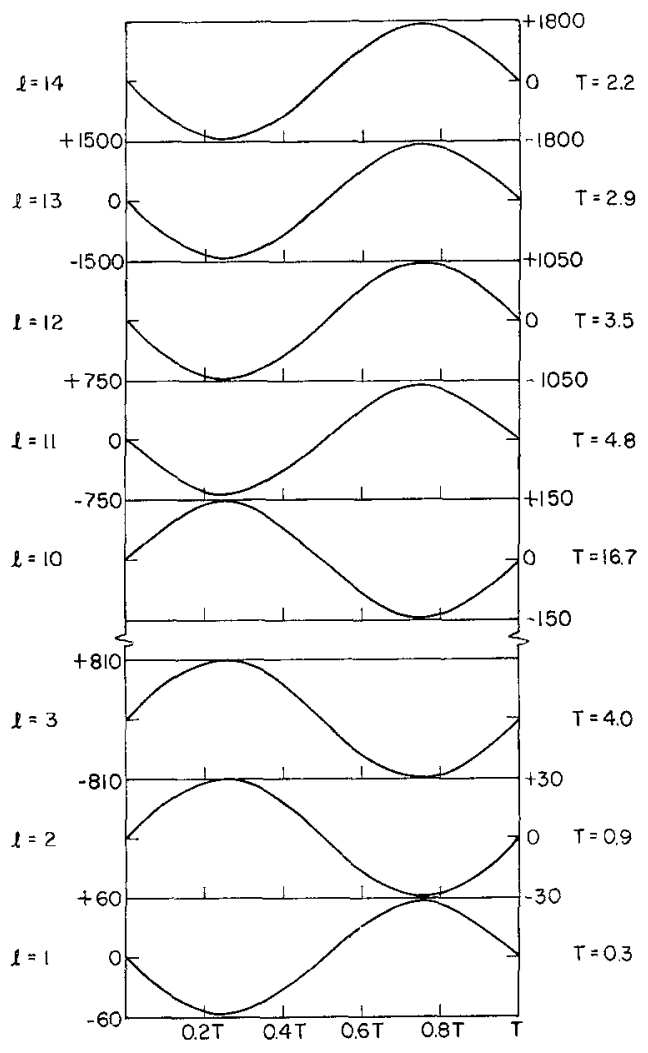

Fig. 7a

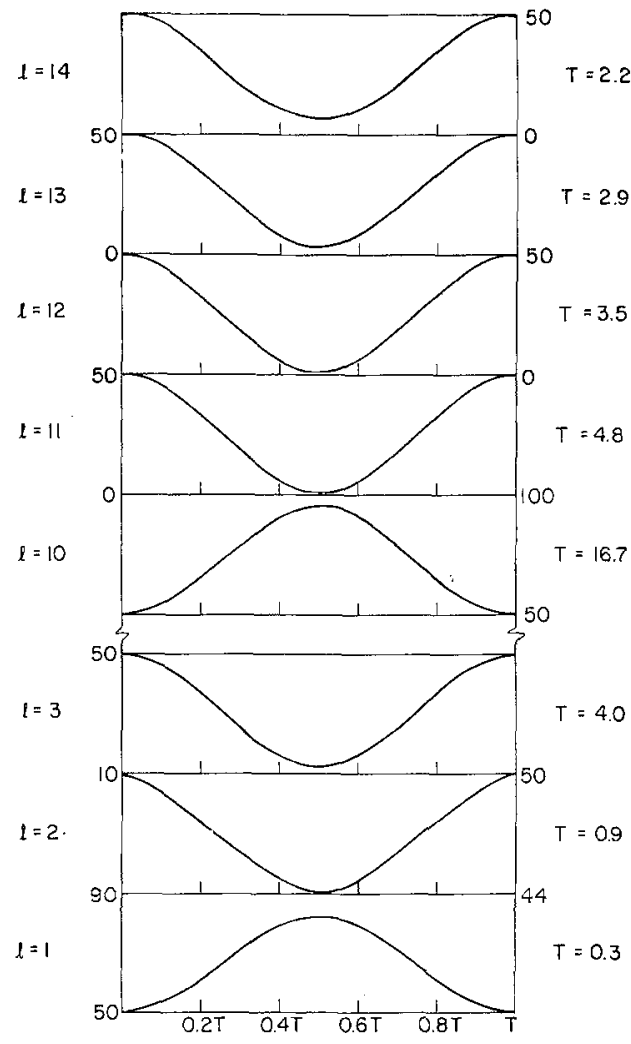

Fig. $7 b$

Figure 7a

The variation of $\overline{\phi v^{*}}$ over one period for stable waves. The wavelength in $10^{3} \mathrm{~km}$ is shown on the left side of the diagram, while the period in days is given to the right. To get the sensible heat transport per unit distance in the unit $\mathrm{kjm}^{-1} \mathrm{sec}^{-1}$ one must multiply the numbers in the figure by $4 P C_{p} / g R \sim 70$.

The ordinate is given alternately on the left and right side of the diagram. $\gamma=0$

Figure $7 b$

The variation of mean flow kinetic energy over one period for stable waves. Arrangement as in fig. 7a. The unit of $K^{*}$ is $\mathrm{kjm}^{-2} \cdot \gamma=0$ 


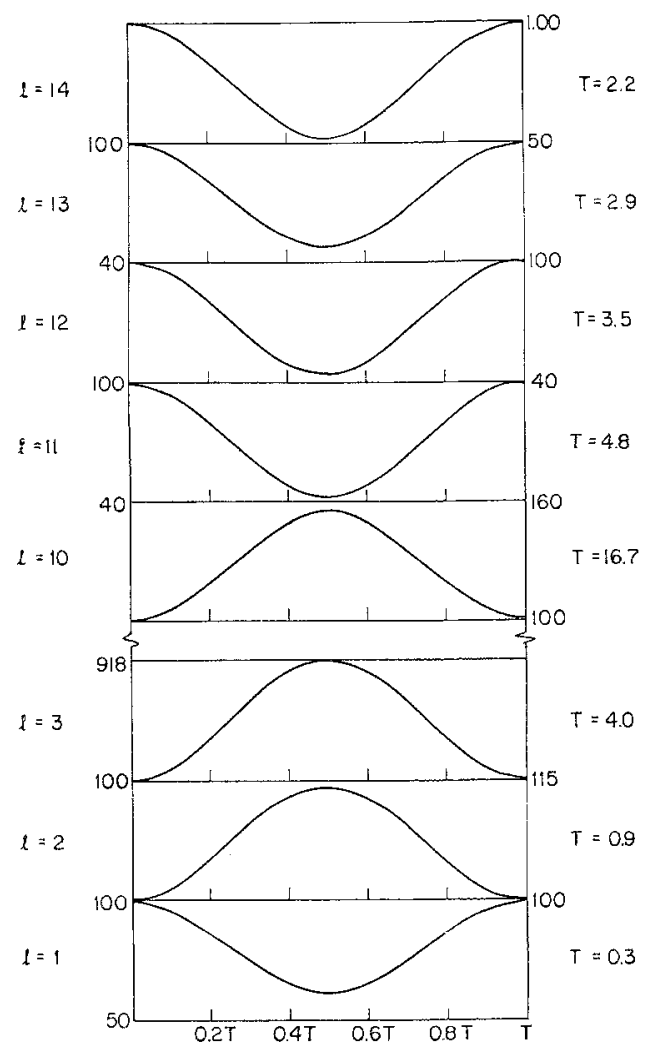

Figure $7 \mathrm{c}$

The variation of the shear flow kinetic energy over one period for stable waves. Arrangement as in fig. 7a. Unit for $K^{\prime}$ is $\mathrm{kjm}^{-2} \cdot \gamma=0$

phase for the short stable waves. This difference is the major difference between short and long stable waves.

We shall next make some comments concerning the magnitude of the energy conversions. For the short stable waves, i.e. $k>q$, we will at any time have the same sign of the conversions. Restricting our attention to $G=0$ we find from the relations $(4.10)$ and (4.11) that

$$
\left|C\left(A_{Z}, A\right)\right|<\left|C\left(A, K^{\prime}\right)\right|<\left|C\left(K^{\prime}, K^{*}\right)\right| .
$$

The major energy conversions will thus take place between the baroclinic and barotropic kinetic energy components. This will mean that the largest oscillations in the energy level will be in the kinetic energy $K^{*}$ as verified by fig. 7a, b, and c, when $k>q$.

When $k<q$ (long waves) we may have either stable or unstable waves. In any case we find that

$$
\left|C\left(A_{Z}, A\right)\right|>\left|C\left(A, K^{\prime}\right)\right|>\left|C\left(K^{\prime}, K^{*}\right)\right|
$$

with the result that the smallest oscillation now occurs in $K^{*}$. Considerable fluctuations 
can take place in both short and long waves. The more important scales for purposes of short range predictions are those which have a period of 1 to 3 days. A typical example illustrated in fig. 7 is $l=14$ where both the mean flow and the shear flow kinetic energies will decrease by a considerable fraction in half a period or about one day. Another example also shown in fig. 7 is $l=3$ with a period of 4 days. In half a period the mean flow energy will increase by about a factor of 9 , while the shear flow will be almost eliminated.

For the unstable waves all energy conversions will increase exponentially at least after a short time, but they will still satisfy (6.12). It is interesting to notice that linear instability theory predicts the inequalities given in (6.12). To the extent we can apply the two level model to atmospheric flow we would expect that the waves of maximum instability will dominate the flow. There is ample evidence that the predictions of unstable waves from baroclinic theory are in qualitative agreement with the observed
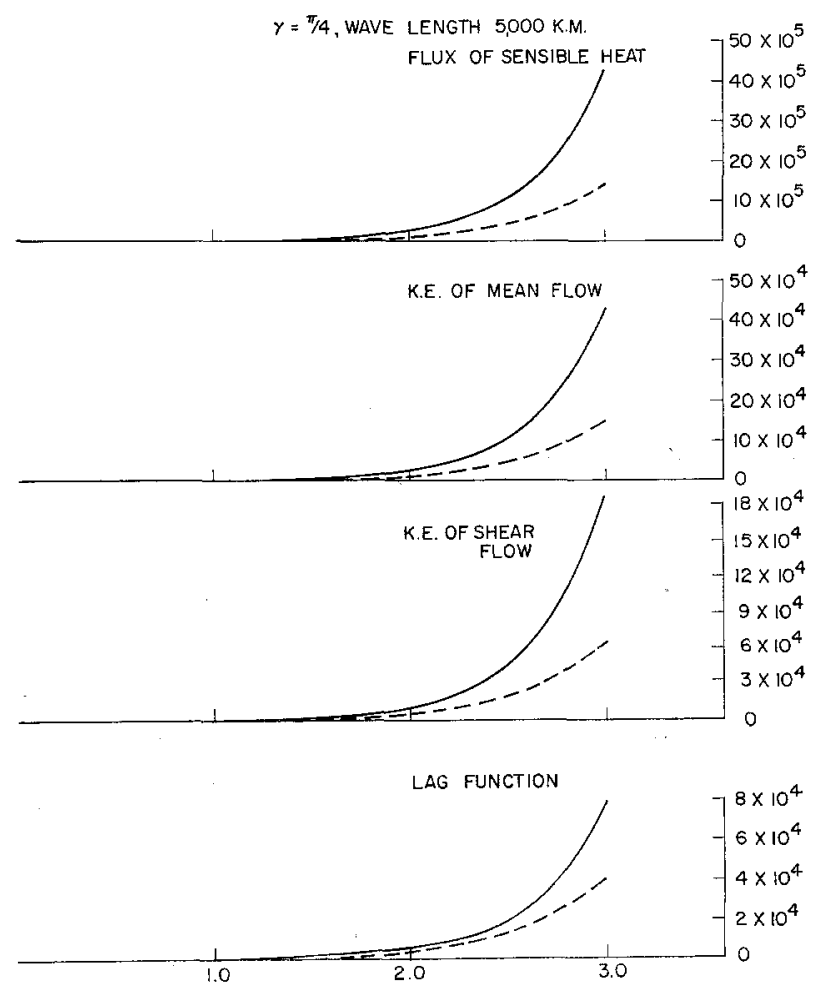

TIME (DAYS)

Figure 8

The variation of $\overline{\phi v^{*}}, K^{*}, K^{\prime}$ and $L$ over 3 days for unstable wave, $l=5$ (in thousands of kilometers). Arrangement and units are as in figs. $7 \mathrm{a}, \mathrm{b}$, and c for $\overline{\phi^{\prime} v^{*}}, K^{*}$, and $K^{\prime}$ respectively. The unit of $L$ is $\mathrm{kjm}^{-2} . \gamma=\pi / 4$. Solid curves are when $G=0$ while dashed curves correspond to $G=3 \times 10^{-6} \mathrm{sec}^{-1}$ 
behavior. Further evidence can be obtained by noting that observational studies (WIIN-NIELSEN [14], fig. 8 and 9) of the energetics of the atmosphere have confirmed (6.12).

The proportionality relations (4.10) and (4.11) are naturally only exact for a given wave number according to the linear theory. It is nevertheless interesting to investigate if there is a tendency for proportionality between the different energy conversions using results of observational studies. The results obtained by KRUEGER, WINSTON and HAINES [7] concerning the relation between $C\left(A_{Z}, A\right)$ and $C\left(A, K^{\prime}\right)$ are significant in this respect. They find that the two energy conversions are correlated with a coefficient of 0.98 and their regression equation shows that $C\left(A_{\mathrm{Z}}, A\right) / C\left(A, K^{\prime}\right)=1.4$. This value may be too large because it is believed that present methods used to evaluate $C\left(A, K^{\prime}\right)$ will underestimate the term. Other studies lead to a value of 1.3 (WIIN-NIELSEN [14]).

Due to the uncertainty in an evaluation of $C\left(A, K^{\prime}\right)$ it becomes interesting to evaluate the ratio $s=C\left(A_{Z}, A\right) / C\left(K^{\prime}, K^{*}\right)$. Averaged data published by WIIN-NIELSEN

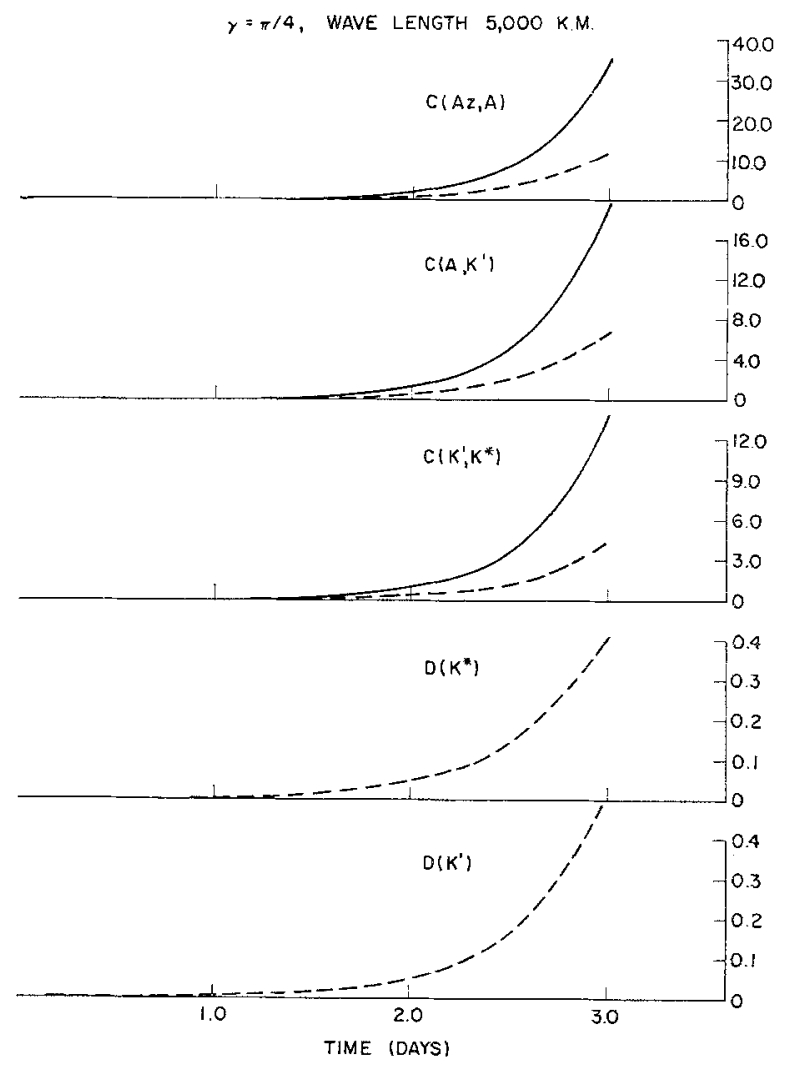

Figure 9

The variation of $C\left(A z_{2} A\right), C\left(A, K^{\prime}\right), C\left(K^{\prime}, K^{*}\right), D\left(K^{*}\right)$ and $D\left(K^{\prime}\right)$ over 3 days in the units: $\mathrm{kjm}^{-2} \mathrm{sec}^{-1}$. Arrangement is as in fig. 8 
[14], gives values of $s=1.34$ for the winter season and $s=1.26$ for summer. The ratio $s$ should be known with greater accuracy than any of the ratios involving $C\left(A, K^{t}\right)$ because the vertical velocity plays a minor role in the determination of $s$. From (4.10) and (4.11) we obtain

$$
q^{2} / k^{2}=s \approx 1.3
$$

from which we can evaluate an effective wavelength. We find $\lambda_{\text {eff }} \approx 3600 \mathrm{~km}$ which is in reasonable agreement with the theory.

We shall next turn our attention to some cases which include friction. In section 4 we have already obtained some information about the behavior of the unstable mode of the form $e^{v t}$. Fig. 8 shows $H, K^{*}, K^{\prime}$ and $L$ as a function of time up to 3 days for unstable waves. The initial conditions were the same as described earlier and $\gamma=\pi / 4$ for this calculation. The marked influence of friction is clearly seen in this figure. After 3 days we have values of $H, K^{*}$ and $K^{\prime}$ which are approximately $\frac{1}{3}$ of the values in the corresponding calculations in which $G=0$, while the lag-function is reduced by a factor 2. Fig. 9 shows the energy conversions and dissipations in a similar arrangement for the same calculation. Note that the inequalities (6.12) are satisfied for both calculations although we have established (6.12) for the case of no friction only.

In the case of stable waves we found pure oscillations in all quantities when friction was excluded from the calculations. We shall next illustrate the influence of friction on stable disturbances. Generally speaking we find that the disturbances approach a steady state characterized by no heat flux which means that the temperature field is in phase with the geopotential field. Fig. 10 and fig. 11 show the heat flux as a function of time for $\lambda=2500 \mathrm{~km}$ and $\lambda=12000 \mathrm{~km}$, respectively. Curves of the heat flux are given for different initial values of $\gamma$. Figs. 12 and 13 show the dissipation $D\left(K^{*}\right)$ for the same values of the wavelength, while figs. 14 and 15 give the dissipation $D\left(K^{\prime}\right)$ as a function of time. It is seen that $D\left(K^{*}\right)$ and $D\left(K^{\prime}\right)$ approach zero in the limit. Figs. 16, 17, 18 and 19 give $K^{*}$ and $K^{\prime}$ as a function of time for two values of the wavelength. For $\lambda=2500 \mathrm{~km}$ we find a ratio of $K^{\prime} / K^{*}$ equal to 0.24 while the same ratio is 0.14 for $\lambda=12000 \mathrm{~km}$. The asymptotic states described above for two different wavelengths are thus steady states characterized by vanishing heat transport and vanishing dissipations, but a certain ratio $K^{\prime} \mid K^{*}$ which depends on wavelengths. In the next section we shall investigate if the ratio $K^{\prime} / K^{*}$ can be deduced from the equations.

\section{Asymptotic Solutions}

The numerical solutions described in section 6 of the paper shows that if we select a point, i.e. a value of $U^{\prime}$ and a value of $l$, in the stable region and to the right of the asymptote $l=2.4$ we find limiting values of the dependent variables which are such that $H, D\left(K^{*}\right)$ and $D\left(K^{\prime}\right)$ tend to zero, while $K^{*}$ and $K^{\prime}$ approach constant values. These asymptotic solutions are only steady state solutions for large values of time. It is of importance to investigate if we can predict the asymptotic values of $K^{\prime} / K^{*}$ or, 


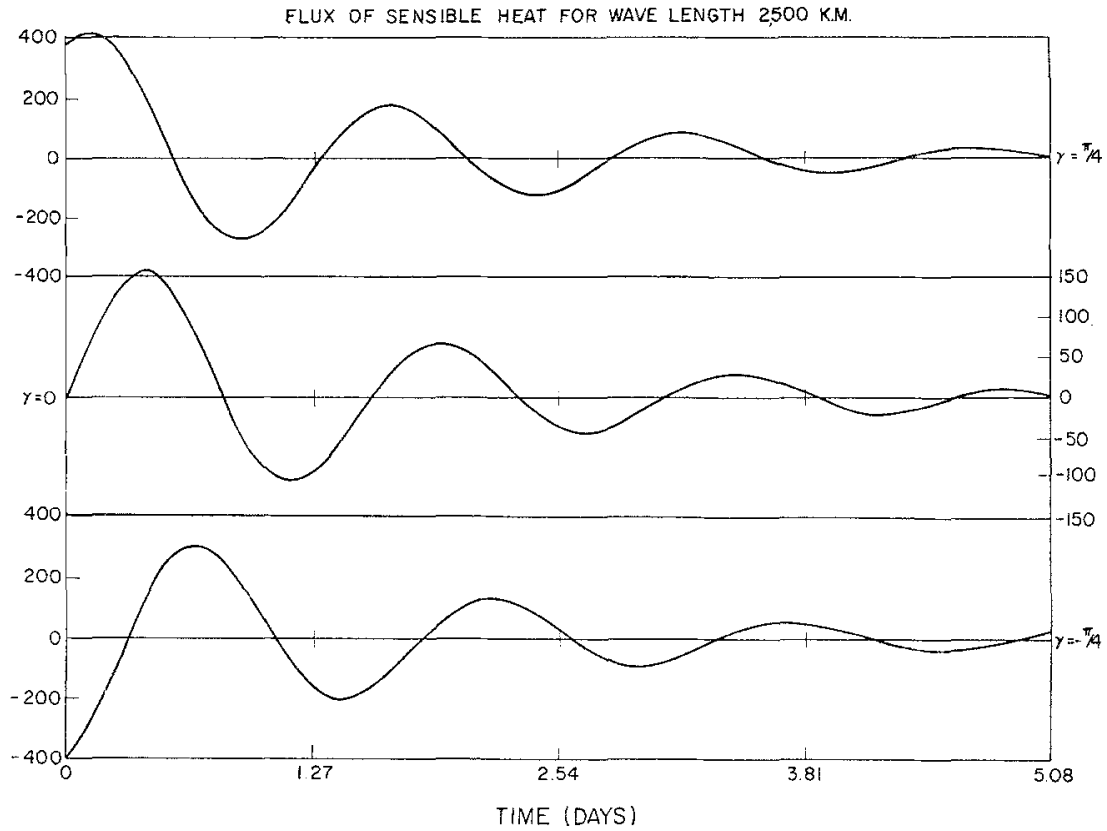

Figure 10

The variation of $\overline{\phi^{\prime} v^{*}}$ over 5.08 days for wavelength $2500 \mathrm{~km}$. for different initial conditions $y=\pi / 4$, $0,-\pi / 4, G=3 \times 10^{-6} \mathrm{sec}^{-1}$. Units: as in fig. $7 \mathrm{a}$

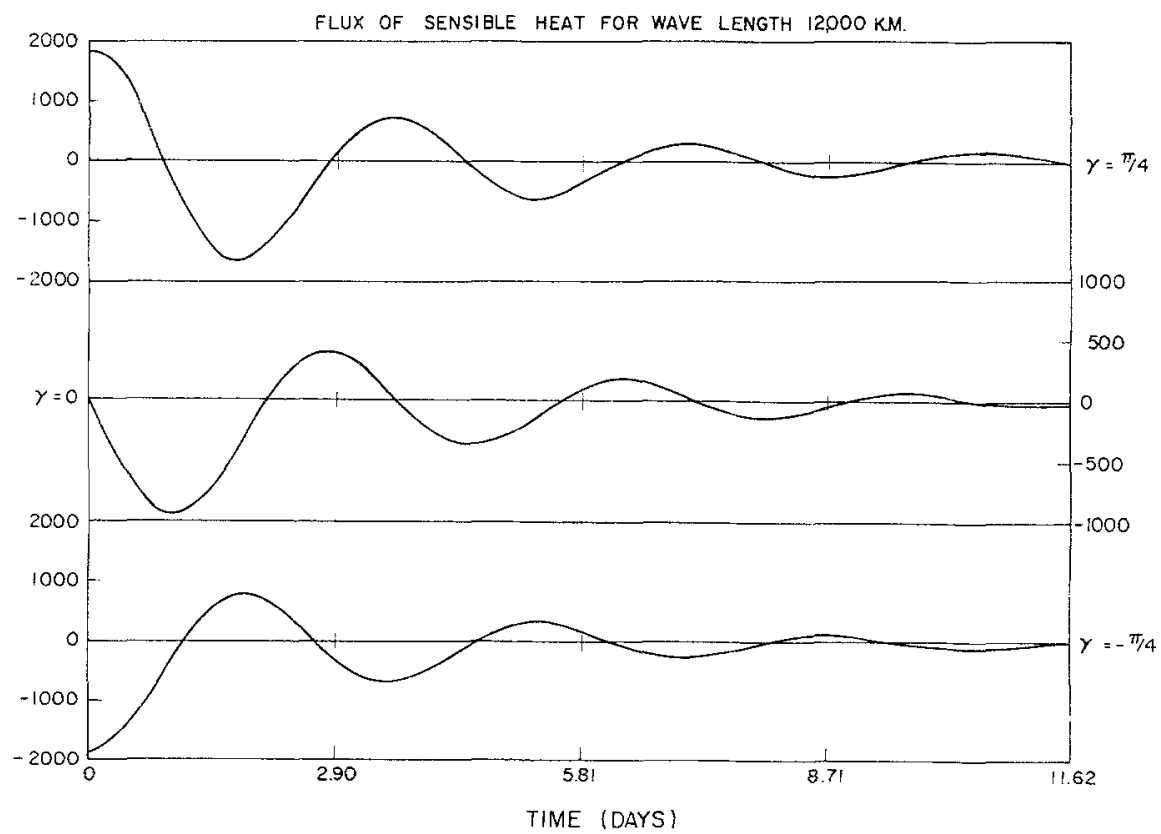

Figure 11

The variation of $\phi^{\prime} v^{*}$ over 11.62 days for wavelength $12000 \mathrm{~km}$. Arrangement and units as in fig. 10 


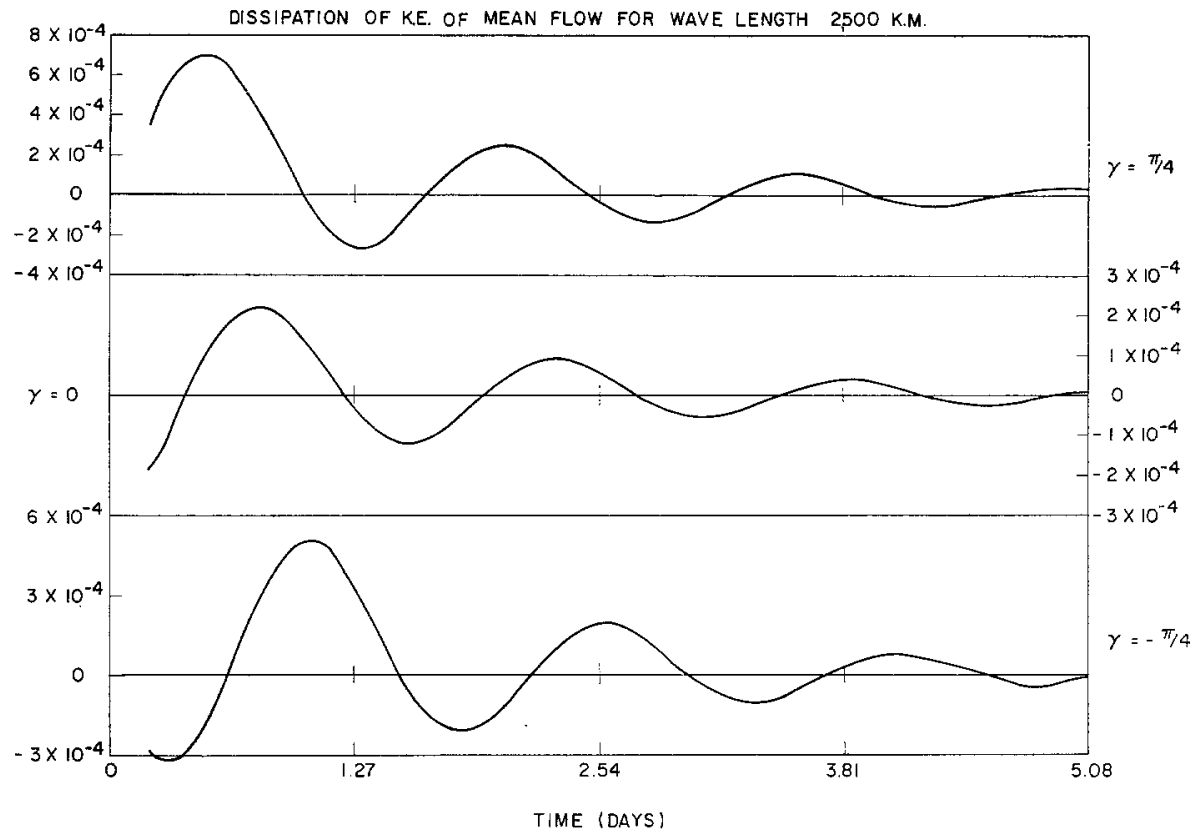

Figure 12

The variation of $D\left(K^{*}\right)$ over 5.08 days for the wavelength $2500 \mathrm{~km}$. Arrangement and units as in fig. 10

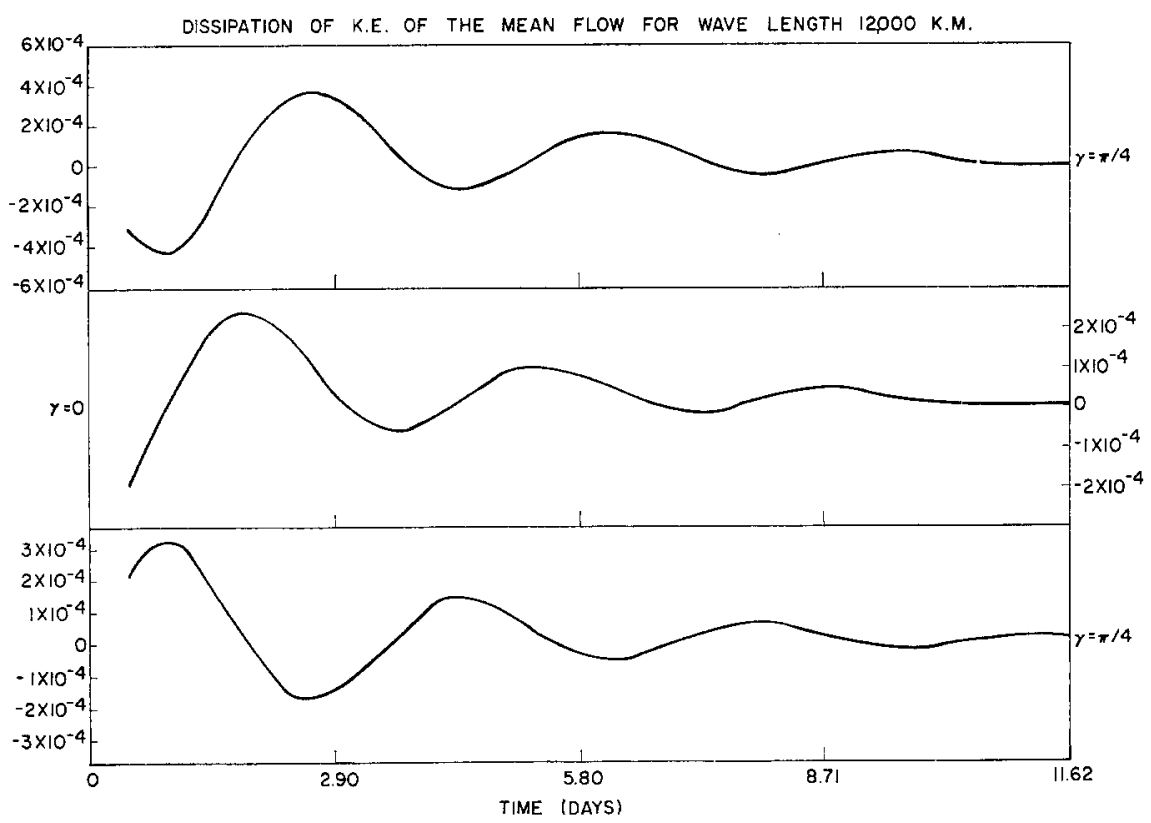

Figure 13

The variation of $D\left(K^{*}\right)$ over 11.62 days for the wavelength $12000 \mathrm{~km}$. Arrangement and units as in fig. 12 


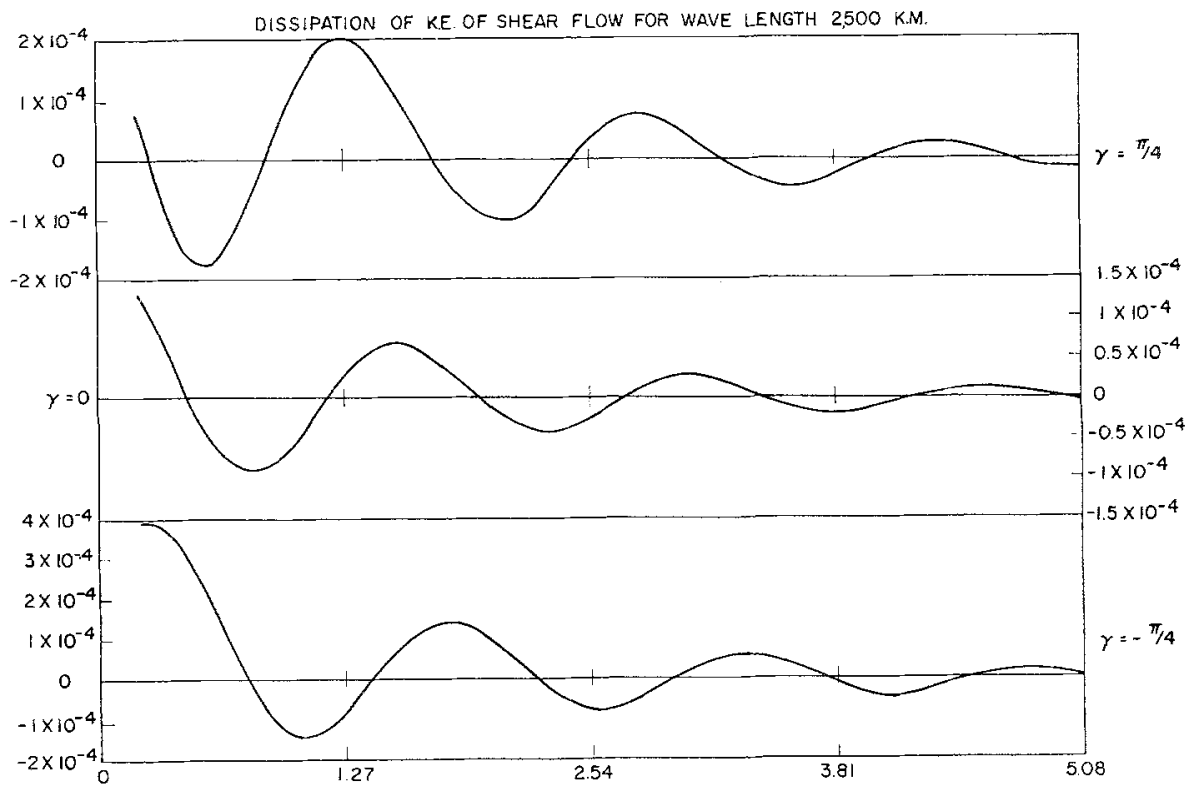

TIME (DAYS)

Figure 14

The variation of $D\left(K^{\prime}\right)$ over 5.08 days for the wavelength $2500 \mathrm{~km}$. Arrangement and units as in fig. 12

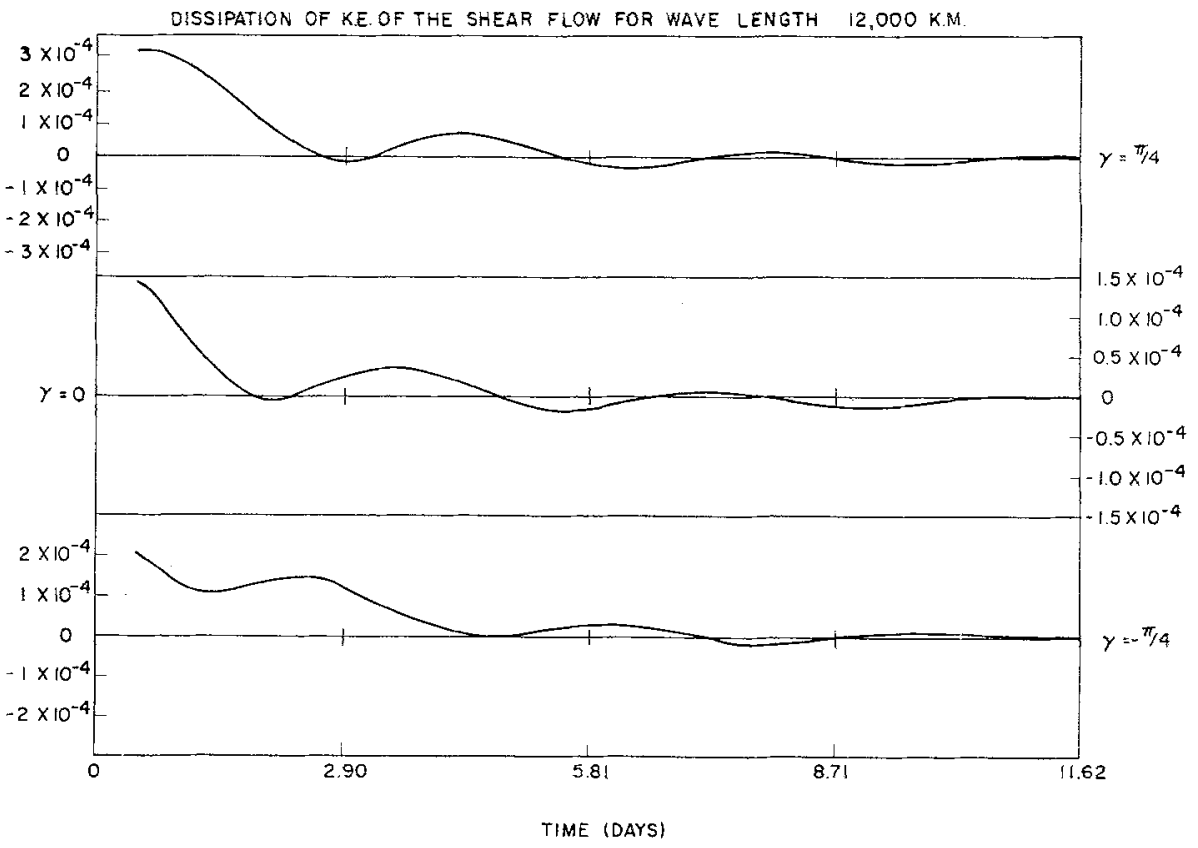

Figure 15

The variation of $D\left(K^{\prime}\right)$ over 11.62 days for the wavelength $12000 \mathrm{~km}$. Arrangement and units as in fig. 12 


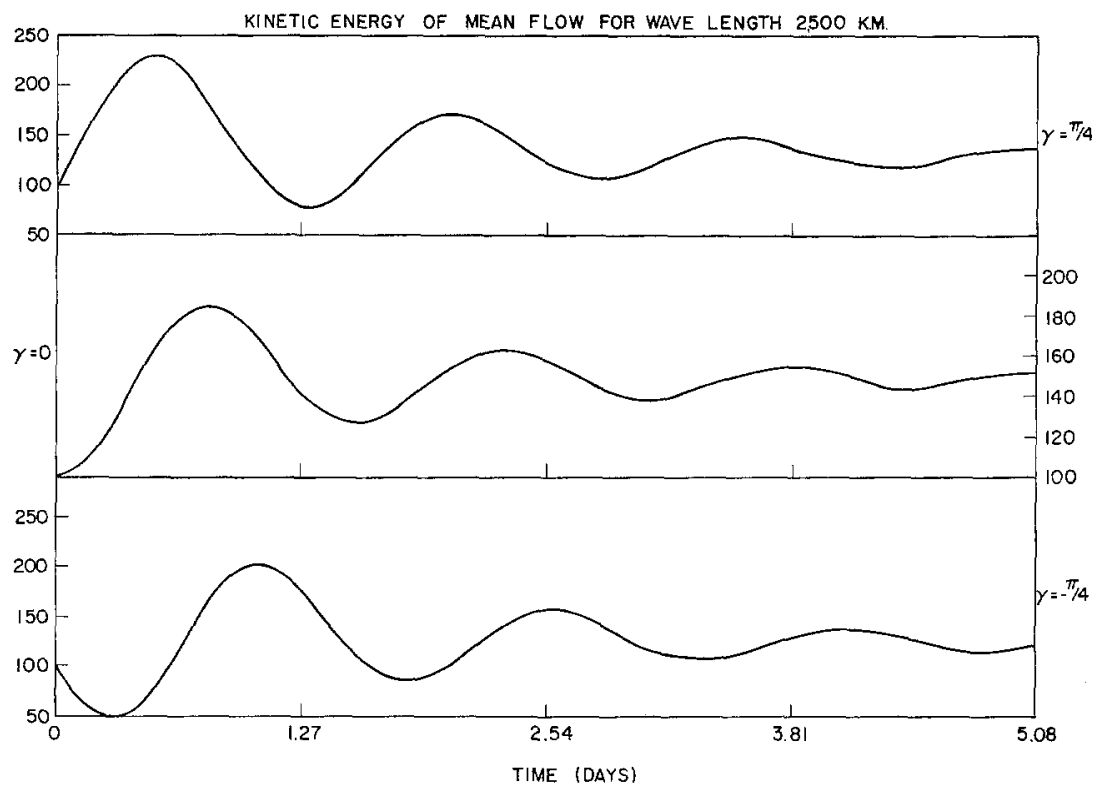

Figure 16

The variation of $K^{*}$ over 5.08 days for the wavelength $2500 \mathrm{~km}$. in the units $\mathrm{kjm}^{-2}$.

Arrangement as in fig. 10

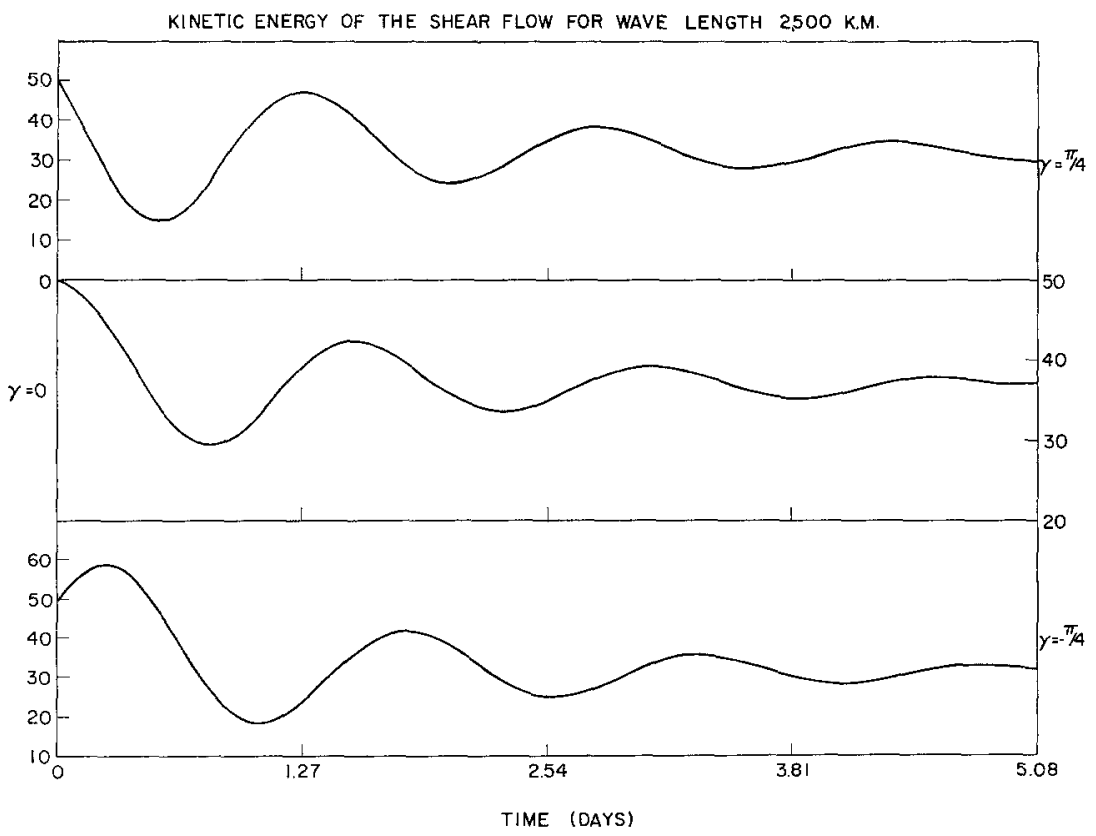

Figure 17

The variation of $K^{\prime}$ over 5.08 days for the wavelength $2500 \mathrm{~km}$. Arrangement and units as in fig. 10 


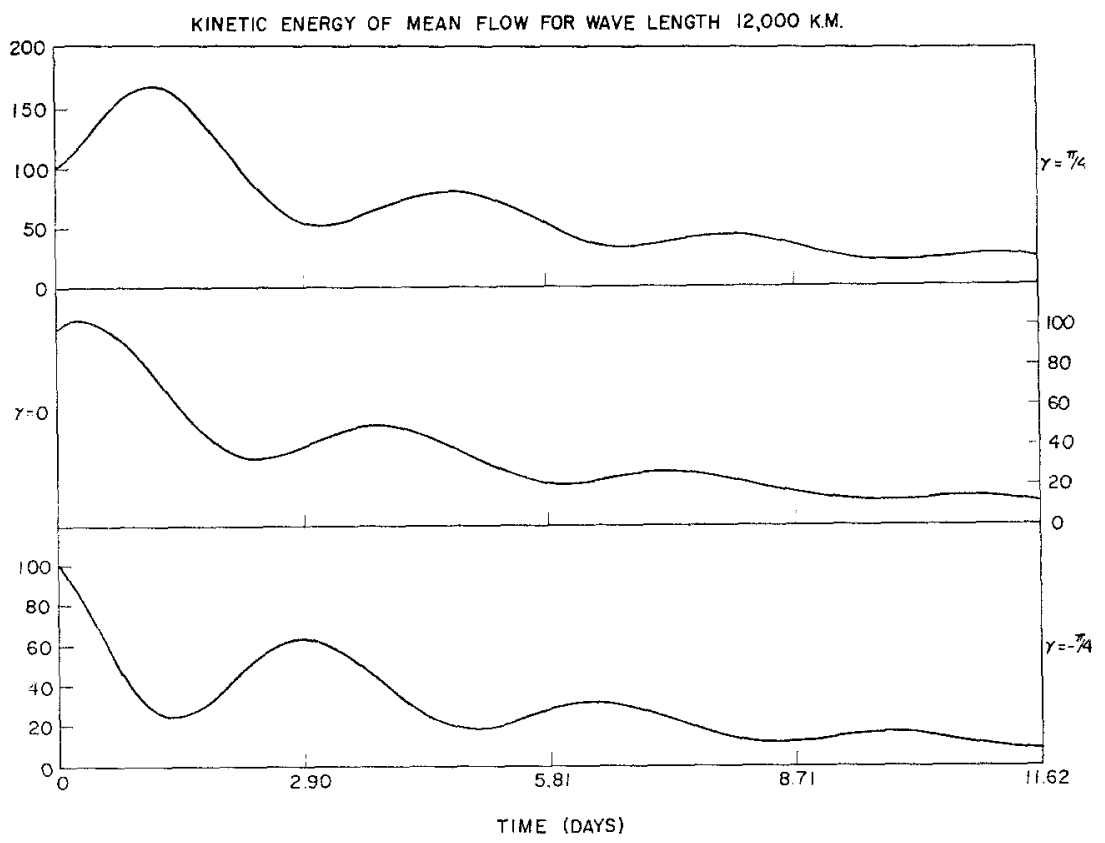

Figure 18

The variation of $K^{*}$ over 11.62 days for the wavelength $12000 \mathrm{~km}$. Arrangement and units as in fig. 10

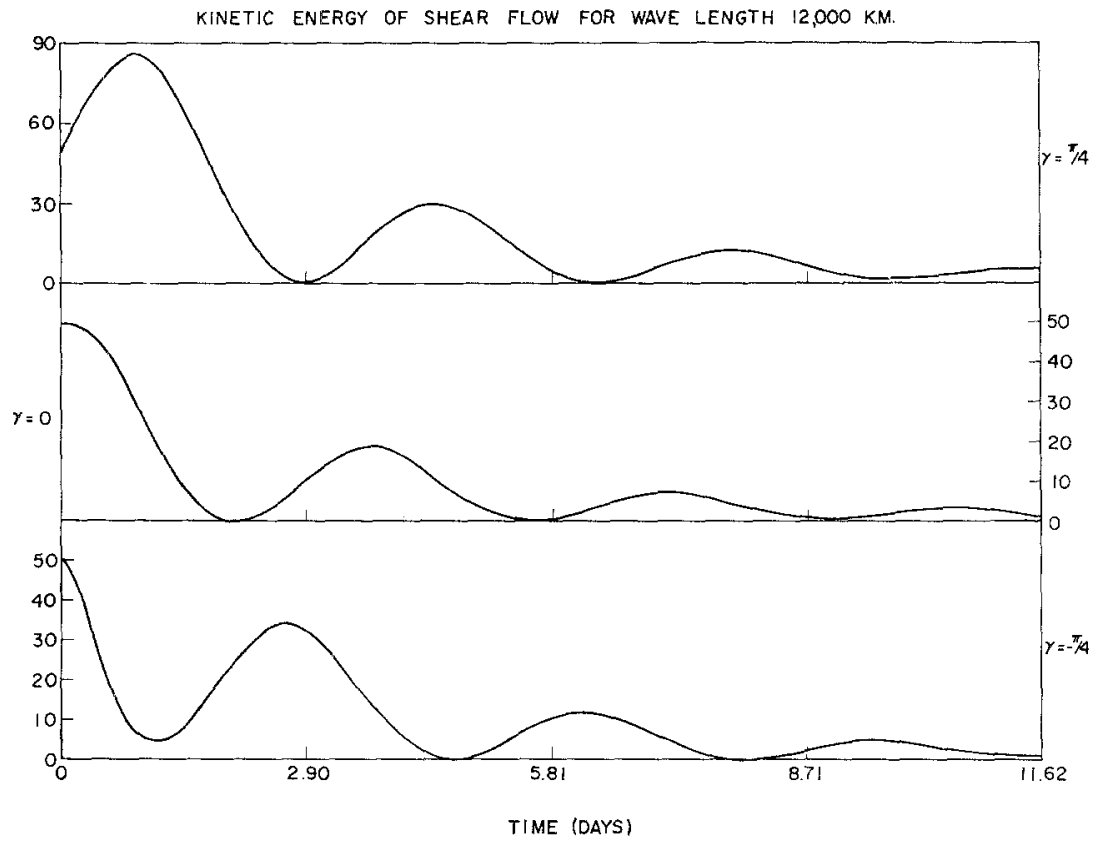

Figure 19

The variation of $K^{\prime}$ over 11.62 days for wavelength $12000 \mathrm{~km}$. Arrangement and units as in fig. 10 
equivalently, $a^{\prime} / a^{*}$. Lacking a solution in closed form of the basic equations (3.5), (3.6), (3.10) and (3.11) with $a=0$, it is difficult to derive the asymptotic behavior of the dependent variables except by numerical integration. However, guided by the fact that the true steady state solutions are independent of friction we may make the hypothesis that the asymptotic solutions are the same regardless of friction. Using this hypothesis we may find the steady state solutions for $G=0$, i.e. no friction, and compare them with the numerical asymptotic solutions.

Setting $G=0$ and $d / d t=0$ in (3.5), (3.6), (3.10) and (3.11) we find $H=0$, which satisfies the first three equations, while (3.11) becomes

$$
-\frac{k^{2}-q^{2}}{k^{2}+q^{2}} U^{\prime} K^{*}+U^{\prime} K^{\prime}-\frac{q^{2}}{k^{2}+q^{2}} C_{R} L=0 .
$$

The phase angle $\gamma=0$, because $H=0$. It follows therefore that $L=\left(p k^{2} / g f_{0}\right) a^{*} a^{\prime}$, and we can therefore express (7.1) in terms of the amplitude ratio $a^{\prime} / a^{*}$. We obtain:

$$
U_{s}^{\prime}\left(\frac{a^{\prime}}{a^{*}}\right)^{2}-\frac{q^{2}}{k^{2}+q^{2}} C_{R}\left(\frac{a^{\prime}}{a^{*}}\right)-\frac{k^{2}-q^{2}}{k^{2}+q^{2}} U_{s}^{\prime}=0 .
$$

The solutions to (7.2) are the amplitude ratios for steady state flow with no friction. According to our hypothesis they are also the amplitude ratios for the asymptotic solutions in the case of friction.

We note first of all that if $a^{\prime} / a^{*}=0.5$ we find from (7.2) that it reduces to the relation (5.10). A steady state solution with friction is therefore also a possible steady state solution without friction. However, the case $G=0$ has other solutions.

All numerical calculations were carried out with $U^{\prime}=20 \mathrm{~m} \mathrm{sec}^{-1}$. Using this value we can compute $a^{\prime} / a^{*}$ as a function of wavelength from (7.2). These values are given in Table 3 as $\left(a^{\prime} / a^{*}\right)^{2}=K^{\prime} / K^{*}$. The values are listed in the first row. It is seen that we get two solutions for each value of $l \geqq 11$. We can now compare these frictionless steady state values of $K^{\prime} / K^{*}$ with the asymptotic values of the same quantity as obtained by numerical integration of the basic equations including friction. The calculations were in all cases continued until we reached a state in which $K^{\prime}$ and $K^{*}$ were approximately constant. The initial values of $K^{\prime}$ and $K^{*}$ were 50 and $100 \mathrm{kjm}^{-2}$, respectively, in all calculations. The second row of Table 3 gives the ratio $\left(K^{\prime} / K^{*}\right)_{a}$

\begin{tabular}{|c|c|c|c|c|c|}
\hline$l$ & 2.5 & 11 & 12 & 13 & 14 \\
\hline$\left(K^{\prime} / K^{*}\right)$ & 0.24 & $\{0.22$ & $\int 0.14$ & $(0.09$ & $\{0.07$ \\
\hline$\left(K^{\prime} / K^{*}\right)_{a}$ & 0.24 & $\begin{array}{r}3.22 \\
0.22\end{array}$ & $\begin{array}{r}5.59 \\
0.14\end{array}$ & $\begin{array}{r}8.61 \\
0.08\end{array}$ & $\begin{array}{r}\{12.4 \\
0.09\end{array}$ \\
\hline$K_{a}^{\prime}$ & 32 & 1.0 & $2 \times 10^{-4}$ & $1.7 \times 10^{-5}$ & $8.5 \times 10^{-6}$ \\
\hline$K^{*}{ }_{a}$ & 131 & 4.6 & $14 \times 10^{-4}$ & $21 \times 10^{-5}$ & $98 \times 10^{-6}$ \\
\hline$T_{a}$ & 30.5 & 96.0 & 69.6 & 57.2 & 49.6 \\
\hline
\end{tabular}

Table 3 
as obtained in these calculations, while the third and fourth row give the asymptotic values of $K_{a}^{\prime}$ and $K_{a}^{*}$. The last row gives the time, measured in days, which was necessary to reach an asymptotic state.

We find good agreement between $\left(K^{\prime} / K^{*}\right)_{a}$ and the smaller values of $\left(K^{\prime} / K^{*}\right)$ given in the first row verifying our hypothesis that asymptotic solutions are independent of friction. The larger values of $\left(K^{\prime} / K^{*}\right)$ listed in the table do not appear in the model with friction. This situation is quite analogous to other hydrodynamic investigations in which several solutions appear in the case with no friction, while the inclusion of friction will select one of the possible solutions. We have furthermore checked our hypothesis by making numerical integrations using a different value of the drag coefficient. The same asymptotic states are reached although it takes longer when we use a smaller value of the drag coefficient. Calculations were made to determine the rate of decay of the amplitude for different values of the drag coefficient, $C_{d}$. It may be noted here that the rate of decay depends not only on $C_{d}$ but also on the scale of motion and the initial conditions. Fig. 20 illustrates a particular case where the $e$-decay time of the

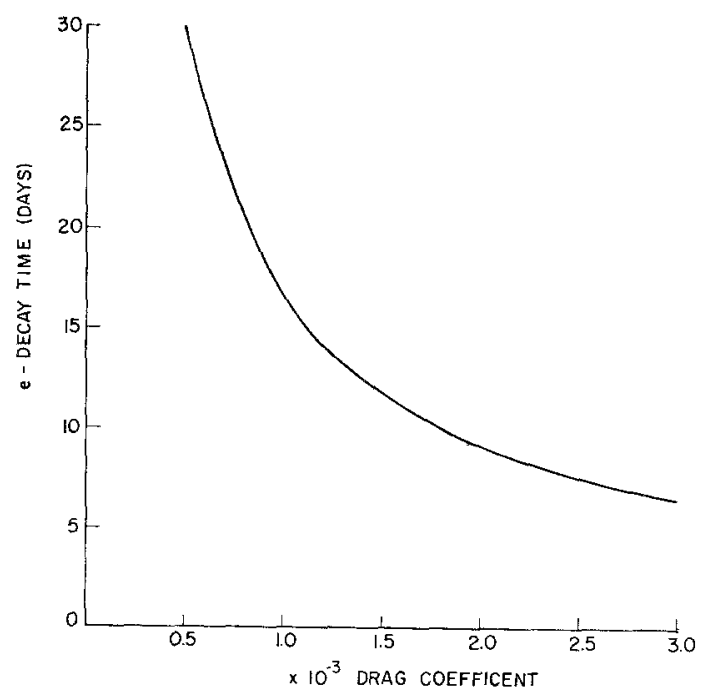

Figure 20

E-decay time of $K^{*}$ in days as a function of drag coefficient for wavelength $12000 \mathrm{~km} . \gamma=\pi / 4$

initial amplitude of $K^{*}$ for wavelength $12000 \mathrm{~km}$ and the same initial conditions as mentioned before except $\gamma=\pi / 4$ is given as a function of $C_{d}$. It is interesting to note here that the $e$-decaying time does not change very much for the values of $C_{d}$ between $2 \times 10^{-3}$ and $3 \times 10^{-3}$ but that it changes very rapidly for smaller values of $C_{d}$. We note finally that the small differences between $\left(K^{\prime} / K^{*}\right)$ and $\left(K^{\prime} / K^{*}\right)_{a}$ found for large $l$ is due to the very low energy levels for these wavelengths approaching the accuracy with which the calculations can be carried out. 


\section{Concluding Remarks}

The main purpose of this investigation has been to investigate the influence of friction and heating on simple baroclinic waves. We have found that there is a change in the region of instability, that friction and heating alters the growth rate of baroclinically unstable waves and that stable waves in general have an asymptotic state independent of friction.

The simple model used in the study is unrealistic because it does not include the effects of topography and non-linearity. It has been the experience in the past that many of the gross features of atmospheric flow can be reproduced from linear models. However, the neglect of topography and the use of the $\beta$-plane approximations will make our results unrealistic for large values of the wavelength. From the results in section 7 it is seen that the ratio $K^{\prime} / K^{*}$ corresponding to the asymptotic solutions become very small when the wavelength is large, and that the heat transport tends to zero in the stable waves. This is in contrast to the observed behavior of the atmosphere on a large scale where it is found that the longest waves have a considerable transport of heat (WIIN-NiELSEN, Brown and DRAKE [13]). Similar differences are also found for very long waves in the values of $\left(K^{\prime} / K^{*}\right)$ when we compare results from the present investigation with observational studies. We notice from Table 3 that $\left(K^{\prime} / K^{*}\right) \approx 0.1$ for the asymptotic solutions, while a ratio of $\left(K^{\prime} / K^{*}\right) \approx 0.3$ is found from the observational study made by WIIN-NIELSEN and DRAKE [15]. On the other hand the energy ratio $\left(K^{\prime} / K^{*}\right)$ which can be derived from fig. 8 shows a value of 0.4 which is in good agreement with the calculations referred to above. While the unstable waves thus show good agreement, we must conclude that topography is of great importance for very long waves in agreement with many earlier studies.

\section{REFERENCES}

[1] J. A. Brown, A Diagnostic Study of Tropospheric Diabatic Heating and the Generation of Available Potential Energy, Tellus 16 (1964), 364-371.

[2] J. G. Charnex, On the General Circulation of the Atmosphere, Rossby Memorial Volume (Rockefeller Press, 1959), 178-193.

[3] A. Eliassen, Simplified Dynamic Models of the Atmosphere Designed for the Purpose of Numerical Weather Prediction, Tellus 4 (1952), 145-156.

[4] R. Fuørtoғт, Some Results Concerning the Distribution and Total Amount of Kinetic Energy in the Atmosphere as a Function of External Heat Sources and Ground Friction, Rossby Memorial Volume (Rockefeller Press, 1959), 194-211.

[5] G. J. Haltiner and E. CaverLy, The Influence of Friction on the Growth and Structure of Baroclinic Waves, Quart. J. Roy. Meteor. Soc. 91 (1965), 209-214.

[6] E. O. Holoparnen, On the Effects of Friction in Baroclinic Waves, Tellus, Vol. 13, 1961 363-367.

[7] A. F. Krueger, J. S. Winston, D. A. Haines, Computations of Atmospheric Energy and its Transformation for the Northern Hemisphere for a Recent Five-Year Period, Monthly Weather Rev. 93 (1965), 227-238.

[8] N. A. Phillips, Energy Transformations and Meridional Circulations Associated with Simple Baroclinic Waves in a Two-level, Quasi-geostrophic Model, Tellus 6 (1954), 273-286.

[9] N. A. PHILlips, The General Circulation of the Atmosphere: A Numerical Experiment, Quart. J. Roy. Meteor. Soc. 82 (1956), 123-165. 
[10] P. D. Thompson, Numerical Weather Analysis and Prediction (MacMillan Company, New York 1961), $170 \mathrm{pp}$.

[11] A. Wirn-Nielsen and J. A. Brown, On Diagnostic Calculations of Atmospheric Heat Sources and Sinks and the Generation of Available Potential Energy, Proceedings of International Symposium on Numerical Weather Prediction, Tokyo (1960), 593-613.

[12] A. WIIN-NIELSEN, On Transformation of Kinetic Energy between the Vertical Shear Flow and the Vertical Mean Flow in the Atmosphere, Monthly Weather Rev. 90 (1962), 311-323.

[13] A. WiIn-Nuelsen, J. A. Brown and M. Drake, Further Studies of Energy Exchange between the Zonal Flow and the Eddies, Tellus, 16 (1964), 168-180.

[14] A. WIIN-NIELSEN, Some New Observational Studies of Energy and Energy Transformations in the Atmosphere, WMO-IUGG Symposium of Research and Development Aspects of Long-Range Forecasting, Proceedings, Technical Note No. 66, WMO, Geneva, Switzerland (1964).

[15] A. WiIN-NIELSEN and M. DRAKe, On the Energy Exchange between the Baroclinic and Barotropic Components of Atmospheric Flow, Monthly Weather Rev. 93 (1965), 79-92.

(Received 25th February 1967) 